\title{
Exenatide induces frataxin expression and improves mitochondrial function in Friedreich ataxia
}

\author{
Mariana Igoillo-Esteve, ${ }^{1}$ Ana F. Oliveira, ${ }^{1}$ Cristina Cosentino, ${ }^{1}$ Federica Fantuzzi, ${ }^{1,3}$ Céline Demarez, ${ }^{1}$ \\ Sanna Toivonen, ${ }^{1}$ Amélie Hu, ${ }^{2}$ Satyan Chintawar, ${ }^{2}$ Miguel Lopes, ${ }^{1}$ Nathalie Pachera, ${ }^{1}$ Ying Cai, ${ }^{1}$ \\ Baroj Abdulkarim, ${ }^{1}$ Myriam Rai, ${ }^{2}$ Lorella Marselli, ${ }^{4}$ Piero Marchetti, ${ }^{4}$ Mohammad Tariq, ${ }^{5}$ \\ Jean-Christophe Jonas, ${ }^{5}$ Marina Boscolo, ${ }^{6}$ Massimo Pandolfo, ${ }^{2}$ Décio L. Eizirik, ${ }^{1,7}$ and Miriam Cnop ${ }^{1,6}$ \\ 'ULB Center for Diabetes Research and 'Laboratory of Experimental Neurology, Université Libre de Bruxelles, Brussels, \\ Belgium. ${ }^{3}$ Endocrinology and Metabolism, Department of Medicine and Surgery, University of Parma, Parma, Italy. \\ ${ }^{4}$ Department of Clinical and Experimental Medicine, University of Pisa, Pisa, Italy. ${ }^{5}$ Pole of Endocrinology, Diabetes \\ and Nutrition, Institute of Experimental and Clinical Research, Université Catholique de Louvain, Brussels, Belgium. \\ ${ }^{6}$ Division of Endocrinology, Erasmus Hospital, Université Libre de Bruxelles, Brussels, Belgium. ${ }^{7}$ Indiana Biosciences \\ Research Institute, Indianapolis, Indiana, USA
}

Friedreich ataxia is an autosomal recessive neurodegenerative disease associated with a high diabetes prevalence. No treatment is available to prevent or delay disease progression. Friedreich ataxia is caused by intronic GAA trinucleotide repeat expansions in the frataxin-encoding FXN gene that reduce frataxin expression, impair iron-sulfur cluster biogenesis, cause oxidative stress, and result in mitochondrial dysfunction and apoptosis. Here we examined the metabolic, neuroprotective, and frataxin-inducing effects of glucagon-like peptide-1 (CLP-1) analogs in in vivo and in vitro models and in patients with Friedreich ataxia. The GLP-1 analog exenatide improved glucose homeostasis of frataxin-deficient mice through enhanced insulin content and secretion in pancreatic $\beta$ cells. Exenatide induced frataxin and iron-sulfur cluster-containing proteins in $\beta$ cells and brain and was protective to sensory neurons in dorsal root ganglia. GLP-1 analogs also induced frataxin expression, reduced oxidative stress, and improved mitochondrial function in Friedreich ataxia patients' induced pluripotent stem cell-derived $\beta$ cells and sensory neurons. The frataxininducing effect of exenatide was confirmed in a pilot trial in Friedreich ataxia patients, showing modest frataxin induction in platelets over a 5-week treatment course. Taken together, GLP-1 analogs improve mitochondrial function in frataxin-deficient cells and induce frataxin expression. Our findings identify incretin receptors as a therapeutic target in Friedreich ataxia.

Conflict of interest: The authors have declared that no conflict of interest exists.

Copyright: ( 2020 , American Society for Clinical Investigation.

Submitted: October 11, 2019 Accepted: December 18, 2019 Published: January 30, 2020.

Reference information: /CI Insight. 2020;5(2):e134221.

https://doi.org/10.1172/jci. insight.134221.

\section{Introduction}

Friedreich ataxia (FRDA) is an autosomal recessive neurodegenerative disease with a prevalence of $1 / 30,000$ in Whites. Most patients are homozygous for a GAA trinucleotide repeat expansion in the first intron of the frataxin-encoding FXN gene; a few are compound heterozygous for an expanded GAA repeat and an $F X N$ loss-of-function mutation (1). Most normal $F X N$ alleles have 8-9 repeats, a few up to 30-35, while expanded alleles contain from 70 to over 1700 repeats that interfere with $F X N$ transcription by heterochromatin silencing (2-4). Longer repeats lead to more severe repression of frataxin expression $(65 \%-95 \%$ decreased compared with healthy controls), such that most residual frataxin in patients with FRDA derives from the allele with the shorter GAA repeat (GAA1), the length of which correlates inversely with age of onset and directly with disease severity $(1,5,6)$. The mitochondrial protein frataxin is involved in iron-sulfur cluster (ISC) biogenesis, and reduced frataxin expression leads to impaired function and/or expression of ISC-containing enzymes, iron accumulation in the mitochondrial matrix, oxidative stress, and mitochondrial dysfunction $(7,8)$. Besides the neurologic manifestations that include progressive gait ataxia, dysarthria, instability, oculomotor abnormalities, and loss of proprioception $(9,10)$, most patients with FRDA develop impaired glucose tolerance or diabetes (11-13) and hypertrophic cardiomyopathy, the latter being the main cause of premature death $(6,10,14)$. Frataxin deficiency causes early loss of large dorsal root ganglia neurons followed by neuronal loss in the cerebellar dentate nucleus and other nervous system regions $(15,16)$, as well as dysfunction and 
apoptosis of insulin-producing pancreatic $\beta$ cells $(13,17)$. Advances in the understanding of FRDA pathophysiology have so far not translated into treatments to prevent, delay, or revert disease manifestations. Antioxidants such as high-dose coenzyme $\mathrm{Q}_{10}$ plus vitamin $\mathrm{E}$ or idebenone (18) failed to show efficacy in clinical trials (19-21). Other approaches tried to enhance frataxin transcription or translation. Interferon- $\gamma(22)$, erythropoietin (23), or epigenetic modifiers such as histone deacetylase inhibitors (HDACis) $(4,24)$ showed promising frataxin induction in in vitro and in vivo models, but the former 2 failed to reach endpoints in clinical trials $(25,26)$ and the latter requires pharmacologic optimization to improve efficacy and reduce toxicity $(27)$.

Incretins are gut hormones secreted in response to food intake. Glucagon-like peptide-1 (GLP-1) is generated by posttranslational cleavage of preproglucagon in enteroendocrine $\mathrm{L}$ cells from the ileum and colon, whereas glucose-dependent insulinotropic polypeptide (GIP) is secreted by duodenal K cells (28). GLP-1 is also produced by preproglucagon neurons in the brainstem (29). It can be released in response to non-nutrient stimuli, including neurotransmitters, neuropeptides, and hormones. GLP-1 and GIP have very short half-lives in the circulation (less than 7 minutes) due to dipeptidyl peptidase IV-mediated degradation. Long-acting GLP-1 analogs, such as exenatide and liraglutide, and dual GLP-1 and GIP agonists have been developed to treat type 2 diabetes $(30,31)$. GLP-1 and GIP receptors are expressed on $\beta$ cells, as well as in brown fat, heart, kidney, and brain (32). GIP and GLP-1 analogs stimulate cAMP formation through binding to their $G$ protein-coupled receptors and activate intracellular signaling pathways that enhance insulin synthesis and glucose-induced insulin secretion and prevent $\beta$ cell apoptosis (33-36). Besides these beneficial effects on $\beta$ cells, the drugs have cardiovascular (37) and neuroprotective actions $(38,39)$. We have previously shown that the cAMP inducer forskolin and incretin analogs [D-Ala $\left.{ }^{2}\right]-G I P$ and exenatide reduce apoptosis in frataxin-deficient $\beta$ cells and neurons in vitro, by decreasing oxidative stress and inhibiting the mitochondrial pathway of apoptosis $(13,17)$. In the course of these experiments, we serendipitously found that cAMP induction enhances frataxin protein expression in vitro in clonal rat $\beta$ cells. Given the potential therapeutic relevance of these findings, the aim of this study was to evaluate the metabolic, neuroprotective, and frataxin-inducing effect of exenatide in in vivo and in vitro FRDA models. We also performed an open-label pilot clinical trial with GLP-1 analogs in patients with FRDA to assess whether the frataxin-inducing effect could be safely obtained in vivo.

\section{Results}

Glucose homeostasis in knockin-knockout mice. The frataxin-deficient knockin-knockout (KIKO; B6.Cg-Fxn ${ }^{\text {tmIMkn }}$ $F x n^{\text {tmIPand }} / \mathrm{J}$ ) mouse has a 230 GAA repeat expansion in 1 Fxn allele and an Fxn knockout in the other (40). Because this mouse has a mild phenotype $(41,42)$, we exposed it to the metabolic stress of high-fat feeding. Wild-type (WT) and KIKO mice were randomized to receive regular $(10 \% \mathrm{kcal}$ fat) or high-fat $\operatorname{diet}(60 \% \mathrm{kcal}$ fat). After 15 weeks, mice were again randomized to receive vehicle (white and gray boxes in Figure 1 and Supplemental Figure 1, respectively; supplemental material available online with this article; https://doi.org/10.1172/jci.insight.134221DS1) or exenatide (see section below for the effect of treatment) for 12 weeks while continuing the diet (Figure 1A and Supplemental Figure 1A). At the end of the dietary intervention, body weight was not different between WT and KIKO mice within each diet group (Figure 1B and Supplemental Figure 1B). There was a trend for KIKO mice to be insulin resistant, with somewhat less glucose lowering during the ITT and higher HOMA-IR (Figure 1, C-E), in keeping with the previously reported insulin resistance gene expression signature in KIKO liver and skeletal muscle (42). High-fat diet induced weight gain in both genotypes $(P<0.001$, Supplemental Figure 1B). As a result, insulin sensitivity decreased 2 -fold in WT mice $(P<0.01$, Supplemental Figure $1, \mathrm{C}-\mathrm{E})$; the decrease was nonsignificant in KIKO mice (that tended to be less insulin sensitive to start with). Fasting glycemia and intraperitoneal (IP) glucose tolerance were similar between genotypes (Figure 1, F-H, and Supplemental Figure 1, F-H). High-fat feeding impaired glucose tolerance of both genotypes $(P<$ 0.001, Supplemental Figure 1, G and H). Insulin levels, the insulinogenic index, and $\beta$ cell function were comparable in KIKO and WT mice; high-fat diet increased fasting and stimulated insulin levels during the IPGTT in both genotypes ( $P<0.05$; Figure $1, \mathrm{I}-\mathrm{K}$; and Supplemental Figure $1, \mathrm{I}-\mathrm{K})$, consistent with an adaptation to insulin resistance. Overall, the metabolic phenotype of KIKO mice is mild compared with the more severe phenotype of patients with FRDA, who are insulin resistant and develop impaired glucose tolerance and diabetes as a consequence of $\beta$ cell failure (13).

Exenatide improves glucose tolerance and $\beta$ cell function in KIKO mice. We next examined the metabolic impact of 12-week exenatide treatment in KIKO and WT mice (red boxes in Figure 1 and Supplemental Figure 1). 


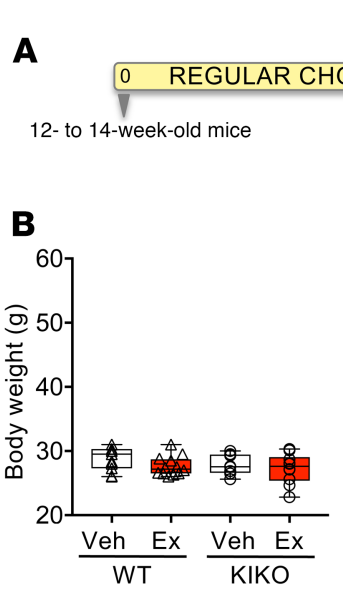

E
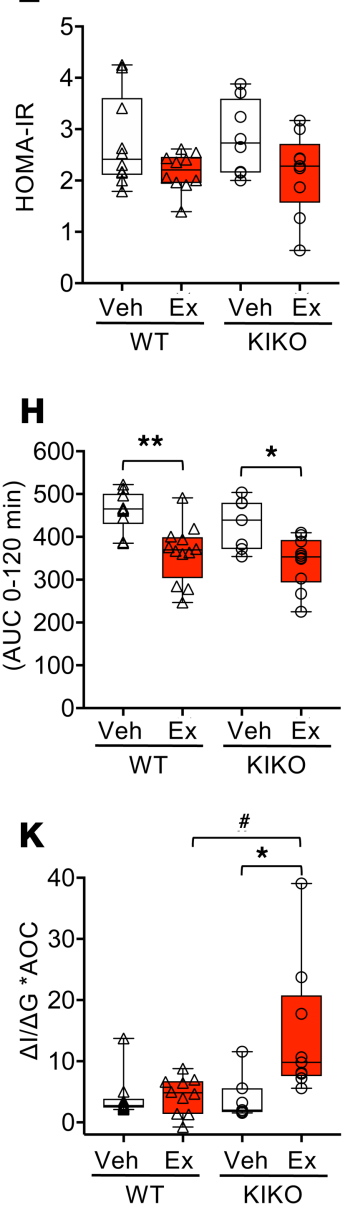

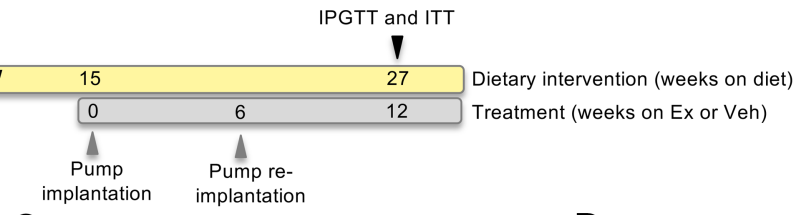

C
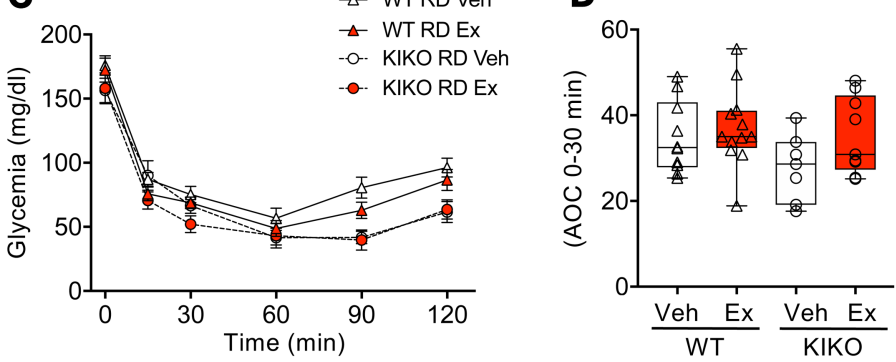

F

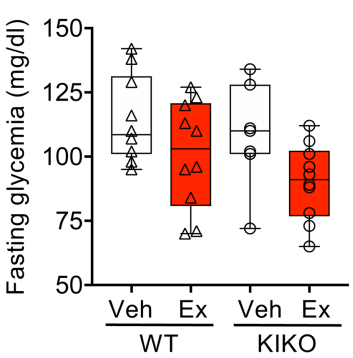

G

I
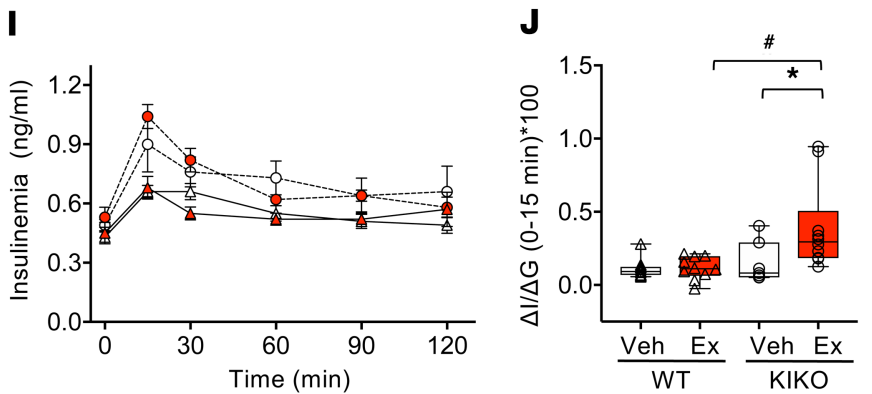

$\mathbf{L}$

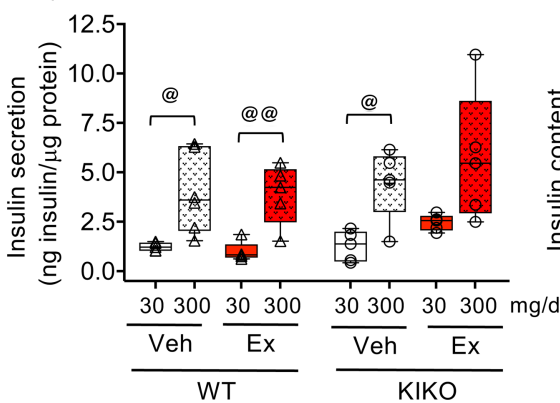

M

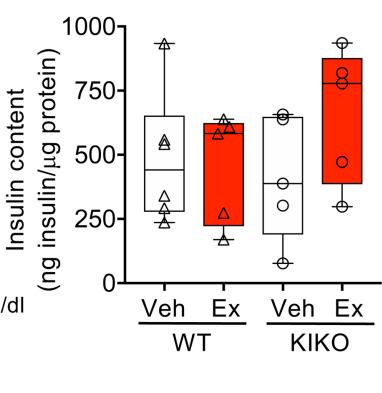

Figure 1. Exenatide improves glucose tolerance and $\beta$ cell function of KIKO mice. (A) WT and KIKO mice, 12 to 14 weeks old, were fed regular chow (RD) for 15 weeks and were then randomized to exenatide (Ex) or vehicle (Veh) administration for 12 weeks ( $n=7-10$ per group). The following assessments were made at the end of the study: (B) Body weight. (C) Glycemia during the insulin tolerance test (ITT). Insulin sensitivity was quantified as area over the curve (AOC) during (D) ITT and (E) homeostatic model assessment of insulin resistance (HOMA-IR). (F) Fasting glycemia (after 16-hour fast). (C) Clycemia, (H) AUC for glucose, and (I) insulin levels during intraperitoneal glucose tolerance test (IPGTT). (J) Insulinogenic index, calculated as incremental insulin divided by incremental glucose $(\Delta \mathrm{l} / \Delta \mathrm{G})$ in the first 15 minutes of the IPGTT. (K) $\beta$ cell function calculated as insulinogenic index corrected for insulin sensitivity. Ex vivo mouse islet glucose-stimulated insulin secretion (L) and insulin content (M) corrected for total protein ( $n=5-6$ per group). Data points correspond to individual mice. The median is shown by a horizontal line in the box plots; 25 th and 75th percentiles are at the bottom and top of the boxes; and whiskers represent minimum and maximum values. ${ }^{*} q<0.05$, KIKO vs. WT; ${ }^{*} q<0.05,{ }^{* *} q<0.01$, and ${ }^{* * *} q<0.001$ Veh vs. Ex; ${ }^{\circledR} q<0.05$, and ${ }^{\circledR} q<0.01300 \mathrm{mg} / \mathrm{dL}$ vs. $30 \mathrm{mg} / \mathrm{dL}$ glucose by Kruskal-Wallis test followed by Benjamini, Krieger, and Yekutieli correction for multiple comparisons or by multiple unpaired 2-tailed $t$ tests (1 per time point, panels $\mathbf{C}, \mathbf{C}$, and I) followed by Holm-Šídák correction for multiple comparisons.

Exenatide did not affect body weight in chow- or high-fat diet-fed mice of either genotype, and it did not change insulin sensitivity (Figure 1, B-E, and Supplemental Figure 1, B-E). Exenatide tended to improve fasting glycemia of chow-fed KIKO mice (Figure 1F), and it improved glucose tolerance of both genotypes (Figure 1, G and H). Exenatide improved the insulinogenic index and $\beta$ cell function of KIKO, but not WT, mice (Figure 1, J and K). A similar beneficial effect was observed in high-fat diet-fed KIKO mice: exenatide improved the insulinogenic index and $\beta$ cell function, but this did not occur in WT mice (Supplemental Figure 1, I-K). Taken together, the GLP-1 analog exenatide improves glucose homeostasis of frataxin-deficient mice through enhanced $\beta$ cell function. 
Exenatide enhances insulin secretion and content of frataxin-deficient islets. Mouse islets were isolated at the end of the experiment to examine insulin content and ex vivo insulin secretion. Exenatide tended to enhance basal insulin secretion and insulin content of KIKO mouse islets (Figure 1, L and M). In islets from high fat-fed KIKO mice, exenatide increased high glucose-stimulated insulin secretion and insulin content (Supplemental Figure 1, L and M). Exenatide did not improve ex vivo insulin secretion or content in islets from WT mice on either diet (Figure 1, L and M, and Supplemental Figure 1, L and M). The enhanced insulin secretion and content in KIKO islets is in keeping with the beneficial in vivo effects of exenatide in KIKO mice.

Exenatide induces frataxin expression in cerebrum and cerebellum of KIKO mice. We previously demonstrated that exenatide, [D-Ala $\left.{ }^{2}\right]$-GIP, and forskolin protect $\beta$ cells from apoptosis (13). In the course of these studies, we unexpectedly observed that these agents upregulate frataxin protein in frataxin-competent clonal rat $\beta$ cells (Supplemental Figure 2). We therefore examined whether exenatide treatment positively modulates frataxin expression in KIKO mouse cerebrum, cerebellum, heart, and islets, the main disease-relevant tissues in FRDA. Twelve-week exenatide treatment tended to induce frataxin protein expression in KIKO mouse cerebrum and cerebellum, as assessed by Western blot and ELISA (Figure 2, A-F). Exenatide did not affect frataxin expression in WT cerebrum and cerebellum (Figure 2, C and F). In keeping with the trend for frataxin induction, exenatide tended to enhance expression of the ISC-containing protein ferrochelatase in KIKO cerebrum and cerebellum (Supplemental Figure 3, A and B). To measure frataxin expression in dorsal root ganglia is technically not possible, so we assessed neuronal cell area instead as a measure of sensory neuron health. Neurons in dorsal root ganglia from KIKO mice were smaller compared with those of WT mice, and this was normalized by exenatide treatment (Figure 2, G and H). The GLP-1 receptor is expressed in islets and heart (Supplemental Figure 3C), but, in contrast with our findings in rat $\beta$ cells (Supplemental Figure 2), exenatide did not affect frataxin protein expression in KIKO or WT islets or heart (Supplemental Figure 3, D-G). The frataxin-inducing effect of exenatide in cerebrum and cerebellum seemed to be independent of transcription because frataxin mRNA expression was unchanged (Supplemental Figure 3, H and I). In summary, in vivo exenatide treatment mildly induces frataxin and ISC-containing ferrochelatase expression in cerebrum and cerebellum of frataxin-deficient mice, and it is protective to sensory neurons in dorsal root ganglia.

Effects of exenatide on FRDA patients' induced pluripotent stem cell-derived $\beta$ cells. To assess the effect of incretin analogs in a patient-relevant model, we generated and differentiated FRDA patients' induced pluripotent stem cells (iPSCs) into pancreatic $\beta$ cells using a 7-stage protocol and neurons (see section below). Fibroblasts were obtained from a 26-year-old male FRDA patient with 1180/980 FXN GAA repeats. His symptoms started before age 10 years with gait instability, followed by progressive trunk and limb ataxia and dysarthria. At onset, tendon reflexes were absent and he had bilateral Babinski sign. He used a wheelchair before age 20; dysarthria, upper limb ataxia, and weakness continued to worsen. He developed optic atrophy and severe hearing loss. He had hypertrophic cardiomyopathy but no heart failure or diabetes. Using episomal vectors, his fibroblasts were reprogrammed into the iPSC line HEL135.2. HEL135.2 iPSCs expressed pluripotency markers OCT4, SSEA4, and TRA1-60; had exogenous transgenes silenced (demonstrated by absent PCR amplification of the episomal vector oriP/EBNA-1 backbone); successfully differentiated into endoderm, mesoderm, and ectoderm germ layers in an embryoid body assay; and had normal 46,XY karyotype (Supplemental Figure 4, A-D). The 7-stage differentiation of HEL135.2 into $\beta$ cells was efficient, resulting in $58 \% \beta$ cells and few $\alpha$ or insulin and glucagon double-positive cells (Figure 3, A and B). Key differentiation markers followed a pattern consistent with pancreatic, endocrine, and $\beta$ cell development, reaching mRNA levels that were overall comparable to clonal insulin-producing EndoC- $\beta \mathrm{H} 1 \beta$ cells and primary human islets (Supplemental Figure 5) and similar to those of control iPSCs differentiating into $\beta$ cells (data not shown). These iPSC- $\beta$ cells had frataxin protein expression levels of around 35\% of healthy control cell line HEL115.6 (Figure 3C). Exenatide treatment mildly induced frataxin expression after 72 hours (Figure 3C). We next measured $\mathrm{NAD}(\mathrm{P}) \mathrm{H}$ autofluorescence in FRDA iPSC- $\beta$ cells, as an indicator of production of metabolic coupling factors by glucose metabolism. Glucose increased $\mathrm{NAD}(\mathrm{P}) \mathrm{H}$ autofluorescence with a larger effect from 0 to $36 \mathrm{mg} / \mathrm{dL}$ than from 36 to $360 \mathrm{mg} / \mathrm{dL}$ glucose, and the mitochondrial uncoupler carbonyl cyanide- $p$-trifluoromethoxyphenylhydrazone (FCCP) (which reoxidizes mitochondrial NADH and NADPH) rapidly reduced $\mathrm{NAD}(\mathrm{P}) \mathrm{H}$ autofluorescence in the aggregates (Figure 3D), indicating that the iPSC- $\beta$ cell mitochondria were metabolically active. $\mathrm{NAD}(\mathrm{P}) \mathrm{H}$ autofluorescence was modestly increased by exenatide (Figure 3D). High glucose induced insulin secretion in iPSC- $\beta$ cells, and a more potent induction was seen with glucose plus forskolin; insulin secretory function was comparable after exenatide treatment (Figure 3E). 


\section{A Cerebrum}

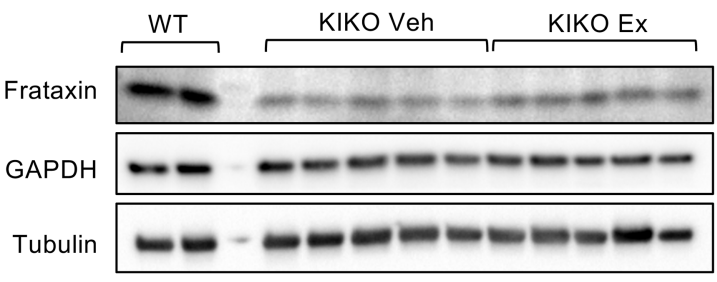

D Cerebellum

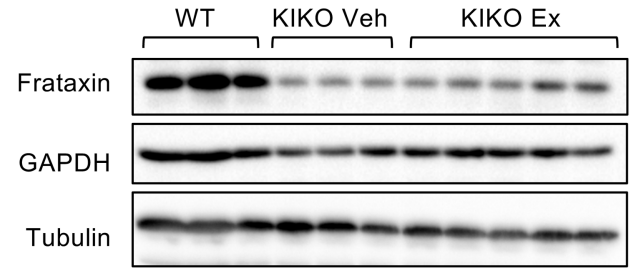

G

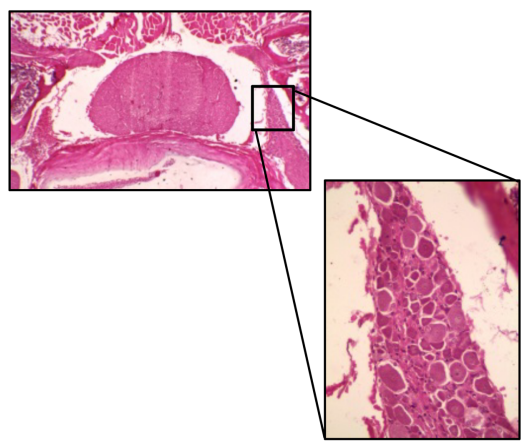

H

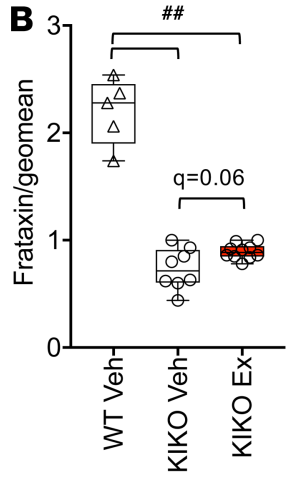

E

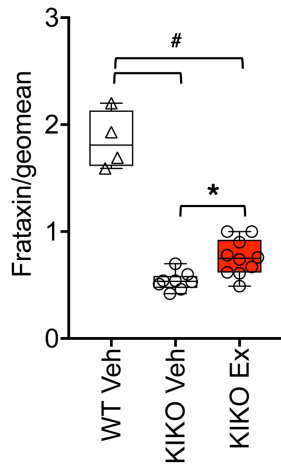

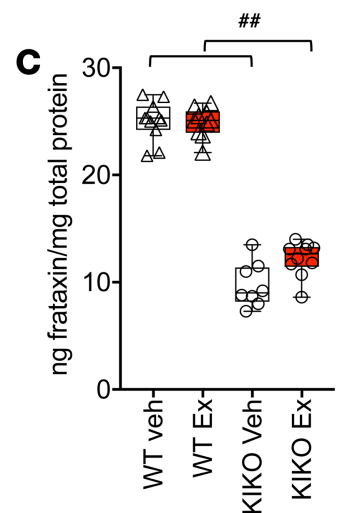

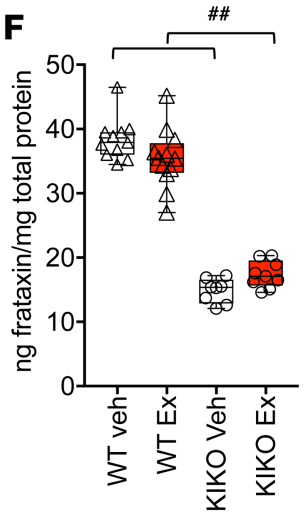

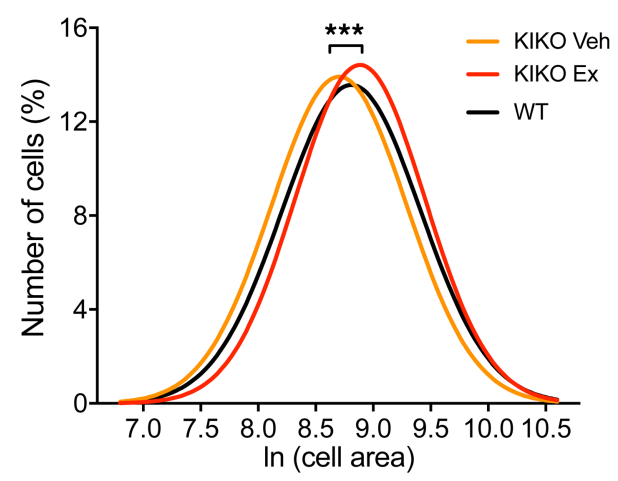

Figure 2. Exenatide induces frataxin expression in KIKO mouse cerebrum and cerebellum and increases dorsal root ganglia neuronal cell area. WT or KIKO mice were treated with saline (Veh) or exenatide (Ex) for 12 weeks. Frataxin protein in cerebrum (A-C) and cerebellum (D-F) was examined by Western blot $(\mathbf{A}, \mathbf{B}, \mathbf{D}, \mathbf{E})$ and mouse frataxin ELISA (C and $\mathbf{F})(n=5-12$ per group). (A and $\mathbf{D})$ Representative Western blot, where each lane corresponds to an independent mouse. (B and E) Densitometric quantification of the blots. Frataxin protein was normalized to the geometric mean of proteins GAPDH and $\alpha$-tubulin (Western blot) or to total protein (ELISA). Data points correspond to individual mice. The median is shown by a horizontal line in the box plots; 25 th and 75 th percentiles are at the bottom and top of the boxes; and whiskers represent minimum and maximum values. ${ }^{*} q<0.05$ Ex vs. Veh; ${ }^{\#} q<0.05$, and ${ }^{\# \#} q<0.01$ KIKO vs. WT by Kruskal-Wallis test followed by Benjamini, Krieger, and Yekutieli correction for multiple comparisons. (G and $\mathbf{H})$ Effect of exenatide on dorsal root ganglia neuronal area. (C) Representative image of a spine section and magnified dorsal root ganglia neurons (original magnification, $\times 4$, and inset, $\times 10)$. (H) Distribution of dorsal root ganglia neuronal cell area (5-6 animals per group) measured by ImageJ (NIH) in WT and KIKO mice treated or not for 12 weeks with exenatide (10-23 pictures per group). The data are shown as a nonlinear fit of cell distribution. ${ }^{* *} P<0.001$ Ex vs. Veh for comparison of the curves by nonlinear regression analysis using extra-sum-of-squares $\mathrm{F}$ test.

Incretin analogs induce frataxin protein in FRDA patients' iPSC-derived neurons. We next examined the effects of incretin analogs on frataxin in patient neurons. We used previously characterized iPSCs from 2 patients with FRDA, FA135 and FA141 (2 clones of each), that carry 800/600 and 900/400 GAA repeats, respectively (43). The iPSCs were differentiated using a protocol that maintains and expands neural progenitors in the form of neurospheres and generates a mixture of neurons and astrocytes $(17,43,44)$. At the end of differentiation the cells expressed low but detectable GLP-1 receptor levels (Supplemental Figure 6A). 


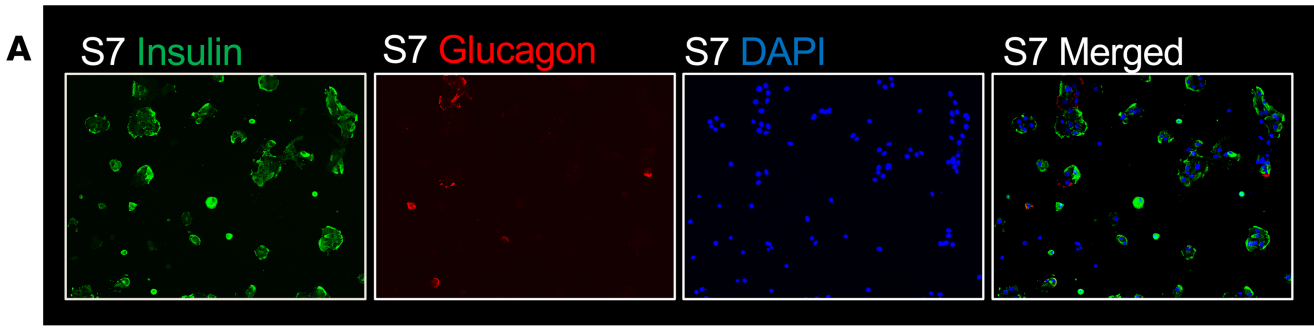

B

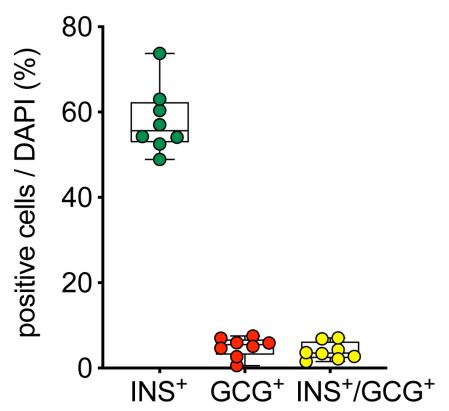

D Glucose Glucose Glucose $0 \mathrm{mg} / \mathrm{dl} 36 \mathrm{mg} / \mathrm{dl} 360 \mathrm{mg} / \mathrm{dl}$

C $24 \mathrm{~h}$

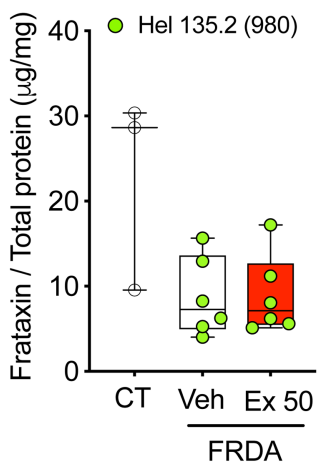

Glucose $360 \mathrm{mg} / \mathrm{dl}$

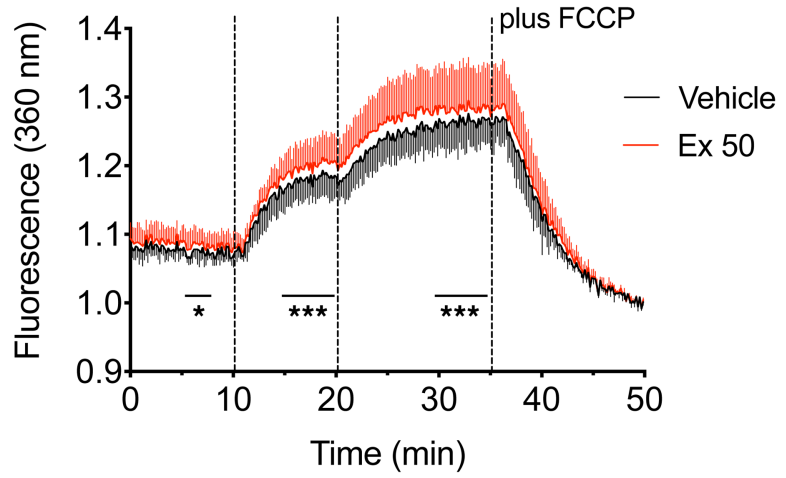

E

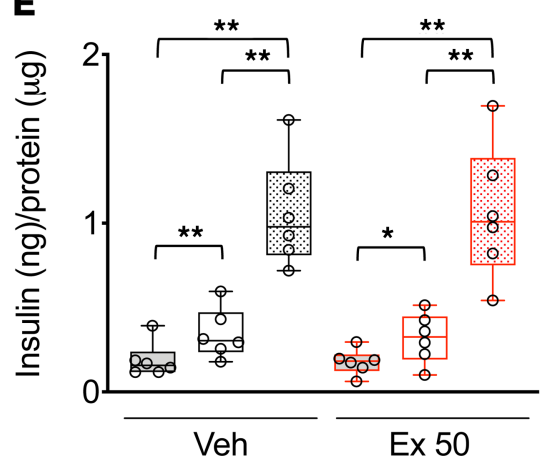

$72 \mathrm{~h}$

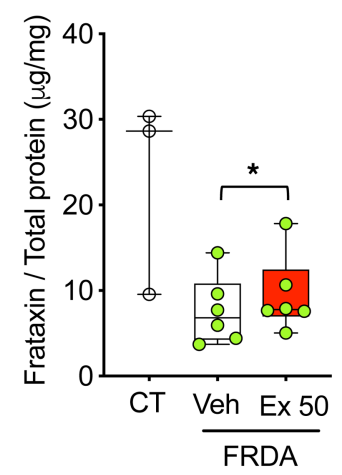

Glucose $30 \mathrm{mg} / \mathrm{dl}$

Glucose $300 \mathrm{mg} / \mathrm{dl}$

Glucose 300 mg/dl plus Forskolin
Figure 3. Frataxin expression and function of an FRDA patient's iPSC-derived pancreatic $\beta$ cells treated with exenatide. FRDA patient iPSCs (HEL135.2, 980 GAA repeats in the smaller $F X N$ allele) were differentiated into $\beta$ cells. (A) Immunofluorescent staining for insulin (shown in green) and glucagon (shown in red) at the end (stage 7) of differentiation. Nuclei are stained with DAPI (blue). (B) Quantification of single or double hormone-positive cells $(n=8)$. (C) Frataxin protein quantified by ELISA in control and FRDA iPSC- $\beta$ cells treated or not (Veh) for 24 or 72 hours with exenatide ( $50 \mathrm{nM}, \mathrm{Ex}$ 50) ( $n=3-6$ per group). ${ }^{*} P<0.05$ Ex vs. Veh by paired 2-tailed $t$ test. (D) $\mathrm{NAD}(\mathrm{P}) \mathrm{H}$ autofluorescence of FRDA iPSC $-\beta$ cells treated or not with exenatide for 24 hours. NAD(P)H data are shown as mean \pm SEM for 6 aggregates from 3 preparations. ${ }^{*} P<0.05$, and ${ }^{* *} P<0.005$ Ex vs. Veh by 2-way ANOVA for matched data followed by Bonferroni's correction for multiple comparisons. (E) High glucose- and high glucose plus forskolin-stimulated insulin secretion by FRDA iPSC- $\beta$ cells treated or not with exenatide for 24 hours $(n=6)$. Data are corrected for total protein. ${ }^{*} P<0.05$, and ${ }^{* *} P<$ 0.01 by paired 2 -tailed $t$ test with Tukey's correction for multiple comparisons. Data points correspond to individual experiments. The median is shown by a horizontal line in the box plots; 25 th and 75 th percentiles are at the bottom and top of the boxes; and whiskers represent minimum and maximum values.

Differentiated cells were then exposed to vehicle, exenatide, [D-Ala $\left.{ }^{2}\right]-G I P$, forskolin, or benzamide HDACi 109 , previously shown to enhance frataxin expression (44). Treatment for 72 hours with 50 or $500 \mathrm{nM}$ exenatide, the GIP analog, or forskolin significantly induced frataxin protein expression, to the same extent as HDAC (Figure 4, A and B). There was no consistent additive effect when exenatide and HDACi were combined (Figure 4, A and B). In line with the mouse data, frataxin mRNA expression was not changed by forskolin or incretin analogs (Supplemental Figure 6B), which is suggestive of posttranscriptional frataxin induction.

The expression of ISC-containing proteins aconitase and $\mathrm{NADH}$ dehydrogenase ubiquinone oxidoreductase Fe-S 3 (NDUFS3) is reduced in iPSC-derived neurons from patients with FRDA (44). 
Similar to HDACi 109 (44), exenatide treatment induced aconitase and NDUFS3 expression in FRDA iPSC-neurons (Figure 4C). Lipoic acid synthase is another ISC-containing enzyme required for the activity and stabilization of pyruvate dehydrogenase (PDH), oxoglutarate dehydrogenase (OGDH), and branchedchain ketoacid dehydrogenase, thereby being an essential cofactor for mitochondrial metabolism (45). The lipoic acid-bound forms of PDH and OGDH are reduced in FRDA iPSC-derived neurons (44). Interestingly, exenatide enhanced lipoic acid-containing $\mathrm{OGDH}$ and $\mathrm{PDH}$ expression (Figure 4C). Collectively, these results show that exenatide induces frataxin expression and ISC availability in patient neurons.

Exenatide induces frataxin and alleviates oxidative stress and mitochondrial dysfunction in FRDA patients' iPSC-derived sensory neurons. We have previously shown that forskolin, [D-Ala $\left.{ }^{2}\right]-G I P$, and exenatide protect frataxin-deficient $\beta$ cells and neurons from apoptosis by decreasing oxidative stress $(13,17)$. Recent developments in iPSC differentiation make it possible to generate functional sensory neurons using a 1-step protocol (46). We implemented this technology to differentiate sensory neurons from FRDA patients' iPSCs FA135 and HEL135 and also used 2 previously characterized control iPSCs, HEL115.6 and HEL46.11 (47, 48). At the end of the 8-day differentiation, most cells expressed sensory neuron-specific markers Brn3a, peripherin, and ISL1 and neuron-specific cytoskeletal marker $\beta$-tubulin III, showing sensory neuron lineage; expression of these markers was confirmed at the mRNA level (Figure 5A and Supplemental Figure 7, A-E). As expected, the patient iPSC-derived neurons expressed low frataxin mRNA and protein compared with healthy control neurons (Figure 5B and Supplemental Figure 7F). In keeping with the findings in iPSC-derived neurons and astrocytes, 72-hour exenatide treatment induced frataxin protein expression in FRDA patient iPSC-derived sensory neurons (Figure 5B), while not modifying frataxin mRNA expression (Supplemental Figure 7F). The patients' sensory neurons had enhanced oxidative stress compared with control cells, and this was reverted by exenatide treatment (Figure 5C). By Seahorse assessment of mitochondrial function, patient iPSC-derived sensory neurons had impaired basal respiration, ATP production, and maximal respiratory capacity compared with control cells (Figure 5, D-G). Exenatide improved these parameters, pointing to improved mitochondrial function (Figure 5, D-G). Taken together, we demonstrate that exenatide induces frataxin, reduces oxidative stress, and improves mitochondrial function in FRDA iPSC-derived sensory neurons, supporting the therapeutic potential of GLP-1 analogs in this neurodegenerative disease.

Exenatide increases frataxin protein expression in patients with FRDA. To evaluate whether GLP-1 analogs induce frataxin expression in patients with FRDA, we performed an open-label pilot trial of 5-week liraglutide or exenatide treatment followed by a 4-week washout (Figure 6A). The primary objective was to assess the frataxin-inducing effect of GLP-1 analogs in peripheral blood mononuclear cells (PBMCs) and platelets, as well as drug safety and tolerability; the secondary objective was to evaluate changes in FRDA clinical rating scales. Sixteen participants, none of them known to have diabetes, were enrolled (baseline characteristics in Table 1).

PBMC and platelet frataxin expression has previously been used as an FRDA biomarker (49, 50): these cells are easily accessible and have GLP-1 receptor expression levels comparable to iPSC-neurons (Supplemental Figure 6A). Because frataxin protein is challenging to quantify (51), frataxin was measured using 3 methods, namely Western blot, lateral flow dipstick immunoassay, and ELISA. As expected, PBMCs and platelets from patients with FRDA showed reduced frataxin mRNA and protein expression compared with unaffected individuals (Supplemental Figure 8, A-C). Frataxin protein levels in patients' PBMCs and platelets were inversely correlated to GAA1 (Supplemental Figure 8, D-F), supporting the use of frataxin protein as a biomarker in these cells. Because of technical issues with platelet and PBMC sample collection and storage resulting in insufficient sample quality, frataxin expression could not be assessed in the liraglutide arm of the trial. In the exenatide arm, frataxin expression was assayed in platelet and PBMC samples. Exenatide led to a modest increase in frataxin protein expression in platelets, as shown using dipstick immunoassay (Figure 6, B and C), Western blot (Figure 6, D and E), and ELISA (Figure 6, F and G). This increase was confirmed when platelet frataxin levels from the 3 assays were averaged (Figure 6, H and I). Frataxin levels in samples from the 5-week exenatide treatment period were well correlated between different assay methods, with the best correlation for dipstick and Western blot (Supplemental Figure 9A). The response to treatment was heterogeneous and varied from no induction in patients with smaller GAA repeat expansions ( 280 and 580 ) to a $20 \%-40 \%$ increase in frataxin protein after 4 weeks of treatment in patients with more than 800 GAA repeats (Figure 6, H and I).

No changes in frataxin protein expression were observed in PBMCs during exenatide treatment (Supplemental Figure 10, A-G), whereas frataxin mRNA expression increased significantly (Supplemental Figure 10, $\mathrm{H}$ and I). It is plausible that PBMCs represent a less suitable cell type than platelets for 
A
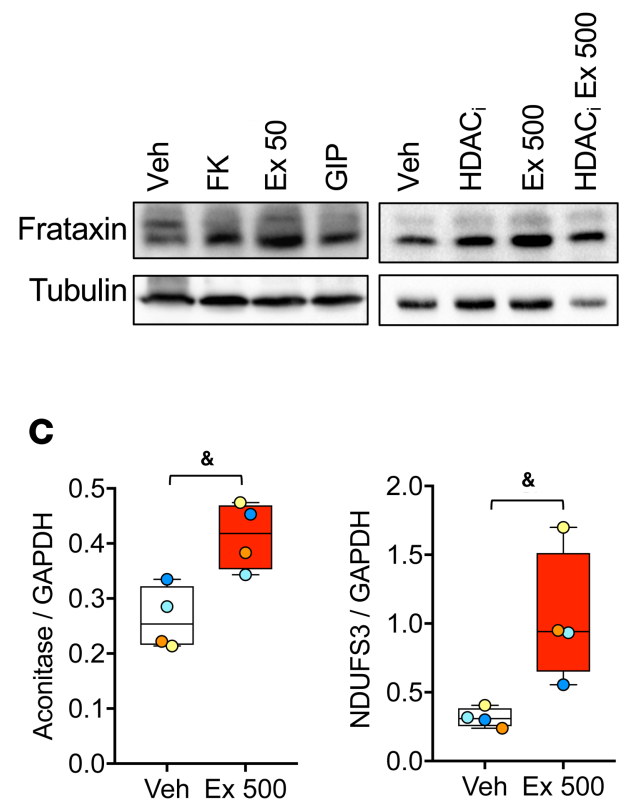

B

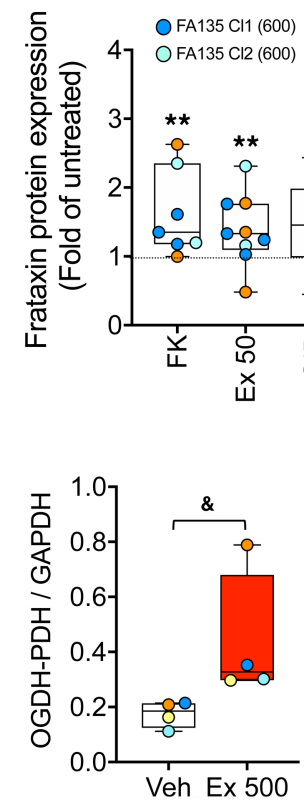

O FA141 Cl1 (400) O FA141 Cl2 (400) ***

Figure 4. Exenatide induces frataxin protein and ISC- or lipoic acid-containing proteins in FRDA patients' iPSC-derived neurons. From iPSC-derived neurons from 2 patients with FRDA (FA135 and FA141), 2 clones ( $\mathrm{Cl} 1$ and $\mathrm{Cl} 2$ ) were treated or not (Veh) for 72 hours with forskolin (20 mM, FK), [D-Ala 2]-GIP ${ }_{1-42}(100 \mathrm{nM}, \mathrm{GIP})$, exenatide (50 or $500 \mathrm{nM}$, Ex), or HDAC $(5$ $\mathrm{mM}$ ) alone or combined with exenatide. (A and B) Frataxin protein expression. (A) Representative Western blot and (B) densitometric quantification of the blots ( $n=6-15$ per condition). The results were normalized to $\alpha$-tubulin and expressed as fold change from untreated samples. (C) Expression of aconitase, NDUFS3, and lipoic acid-containing forms of OCDH and PDH was examined by Western blot and normalized to GAPDH $(n=4)$. Data are represented using box plots. The horizontal line in the box corresponds to the median; 25th and 75th percentiles are at the bottom and top of the box; and whiskers indicate minimum and maximum values. Data points represent independent experiments. Patients and clones are shown in different colors; the smaller GAA expansion size in $F X N$ is shown in brackets. ${ }^{*} q<0.05,{ }^{* *} q<0.01$, and ${ }^{* * *} q$ $<0.001$ treated vs. untreated by Kruskal-Wallis test followed by Benjamini, Krieger, and Yekutieli correction for multiple comparisons; ${ }^{\circledR} P<0.05$ Ex 500 vs. Veh, by paired 2-tailed $t$ test.

frataxin protein measurement, as suggested by the poorer correlation between different assays to measure frataxin in PBMCs (Supplemental Figure 9B) compared with platelets (Supplemental Figure 9A). The sensitivity to detect small changes in frataxin protein may thus be less in PBMCs.

No serious adverse events occurred during the study. The most common side effects were nausea and decreased appetite (Table 2), as described for exenatide or liraglutide therapy at the rapidly titrated doses. The GLP-1 analogs led to an average of $1.5 \mathrm{~kg}$ body weight loss, which was regained after washout (Table 3 ). In this nondiabetic population of patients with FRDA, HbAlc dropped by $0.2 \%$ over the 5 -week treatment period (Table 3). There was no change in the neurologic deficit of patients after 5-week exenatide or liraglutide treatment, as assessed by the Scale for the Assessment and Rating of Ataxia, Inventory of Non-Ataxic Symptoms, and Activities of Daily Living scales (Table 3)

\section{Discussion}

There are currently no disease-modifying therapies for FRDA, and treatment remains symptomatic and supportive. We have previously demonstrated that cAMP induction prevents apoptosis and reduces oxidative stress in frataxin-deficient $\beta$ cells and neurons $(13,17)$. In this study, we set out to investigate the therapeutic potential of GLP-1 analogs for FRDA using in vitro and in vivo disease models. The data show that 12 -week exenatide treatment in vivo improves $\beta$ cell insulin secretory function in KIKO mice, mildly induces frataxin and ISC-containing protein expression in cerebrum and cerebellum, and confers sensory neuroprotection in dorsal root ganglia. In vitro in FRDA patients' iPSC-derived neurons and sensory neurons, generated using 2 differentiation protocols, exenatide induces frataxin, induces ISC availability, reduces oxidative stress, and improves mitochondrial function. Based on these promising preclinical findings, we assessed the frataxin-inducing effect in a pilot study in patients with FRDA and 
A

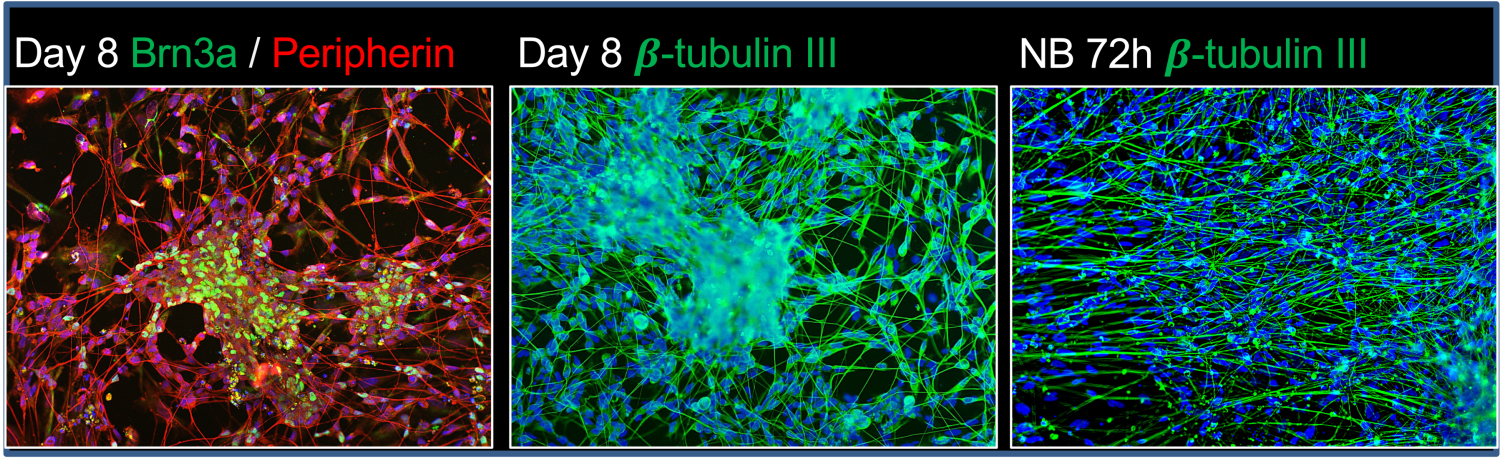

B

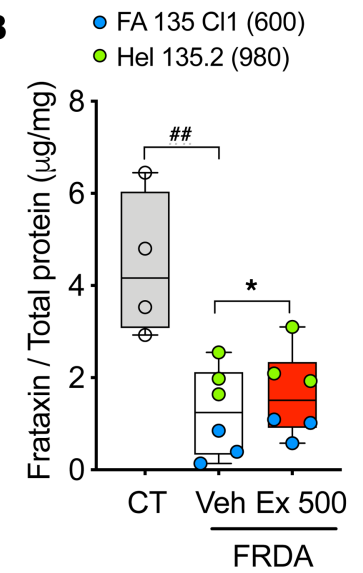

E

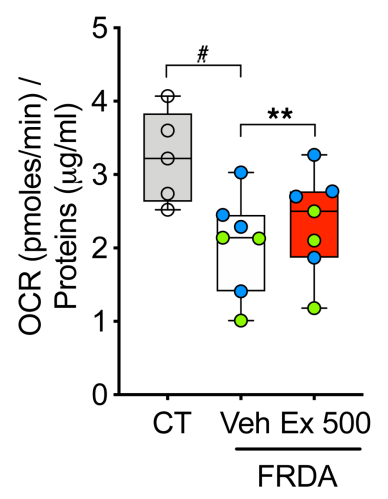

C

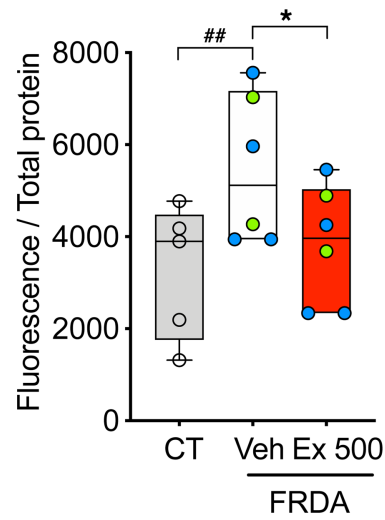

$\mathbf{F}$

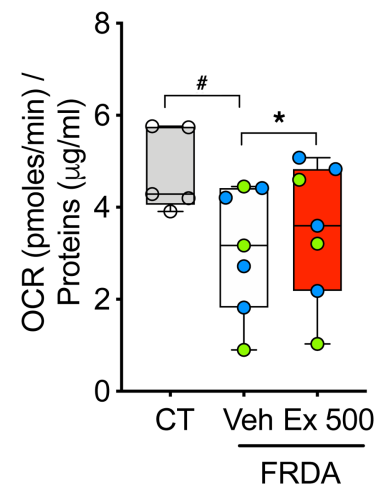

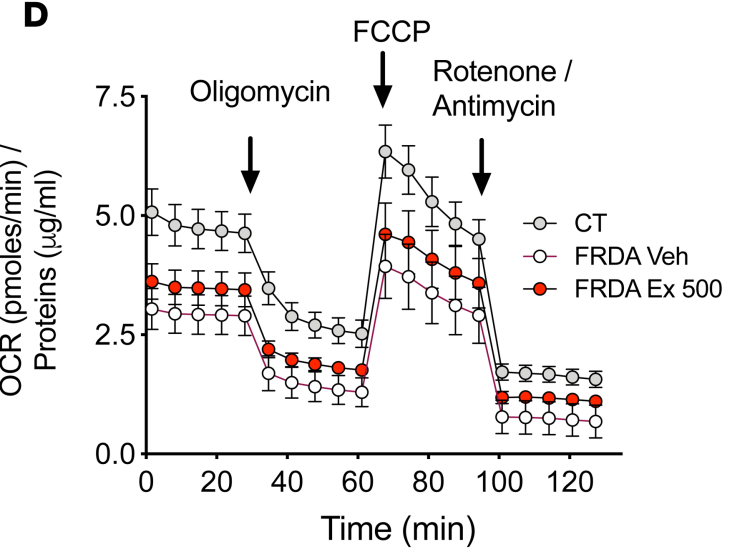

G

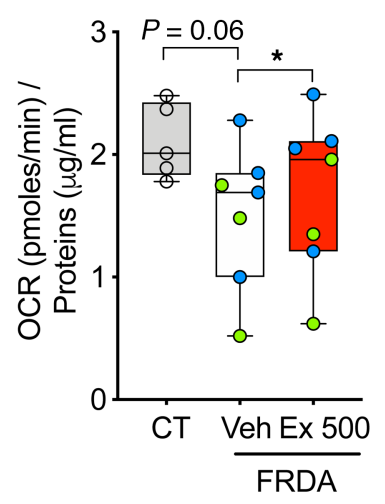

Figure 5. Exenatide alleviates oxidative stress and mitochondrial dysfunction in FRDA patients' iPSC-derived sensory neurons. Sensory neurons were differentiated from iPSCs from 2 healthy controls (CT1 and CT2) and 2 patients with FRDA (FA135 CI1 and HEL135.2). At the end of differentiation cells were treated for 72 hours with PBS (Veh) or exenatide (Ex, $500 \mathrm{nM}$ ) in neurobasal medium. (A) Representative immunofluorescence of CT1 cells during sensory neuron differentiation (images are representative of 7 similar experiments; more images are shown in Supplemental Figure $7 \mathrm{~A}$ ). Pictures were taken at original magnification $\times 20$. Expression of sensory neuron markers Brn3a and peripherin was examined at day 8 and neuron-specific cytoskeleton marker $\beta$-tubulin III at day 8 and after 72 -hour culture in neurobasal medium (NB). (B) Frataxin protein expression was analyzed by ELISA and normalized to total protein ( $n=4-6$ per group). (C) Oxidative stress was measured with hydroxyphenyl fluorescein (HPF) probe. (D-G) Mitochondrial respiration was assessed by XFp Extracellular Flux Analyzer (Seahorse, $n=5-7$ per group). (D) Oxygen consumption rate (OCR) profiles of control and FRDA patients' sensory neurons in basal condition and after injection (arrows) of the ATP synthase inhibitor oligomycin, the mitochondrial uncoupler FCCP, and the metabolic poisons rotenone and antimycin A. (E-G) OCR measures were used to calculate basal respiration, ATP production, and maximal respiratory capacity. The horizontal line in the box corresponds to the median; 25 th and 75 th percentiles are at the bottom and top of the boxes; and whiskers indicate minimum and maximum values. Data points represent independent experiments. Patients are shown in different colors; the smaller GAA expansion size in FXN is shown in brackets. ${ }^{P}<0.05$, and ${ }^{\# \#} P<0.01$ CT vs. FRDA Veh by unpaired 2 -tailed $t$ test; ${ }^{*} P<0.05$, and ${ }^{* *} P<0.01$ FRDA Veh vs. FRDA Ex 500 by paired 2 -tailed $t$ test. 
A

\begin{tabular}{|c|c|c|c|c|}
\hline Baseline (2 w) & \multicolumn{3}{|c|}{ Exenatide treatment (5 w) } & Washout (4 w) \\
\hline $\begin{array}{c}\text { Visits } 1 \text { and } 2 \\
\text { Clinical evaluation } \\
\text { and blood sampling }\end{array}$ & $\begin{array}{c}\text { Starting dose } \\
(1 \mathrm{w}) \\
5 \mu \mathrm{g} \mathrm{BID}\end{array}$ & $\begin{array}{c}\text { Standard dose } \\
(2 \mathrm{w}) \\
10 \mu \mathrm{g} \mathrm{BID}\end{array}$ & $\begin{array}{c}\text { Standard dose } \\
(2 \mathrm{w}) \\
10 \mu \mathrm{g} \mathrm{BID}\end{array}$ & $\begin{array}{l}\text { Weeks } 7 \text { and } 9 \\
\text { Clinical evaluation } \\
\text { and blood sampling }\end{array}$ \\
\hline
\end{tabular}

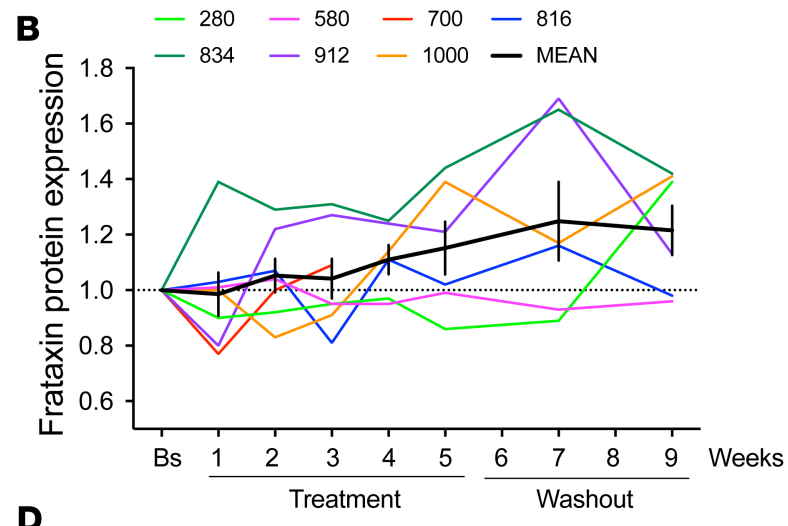

D

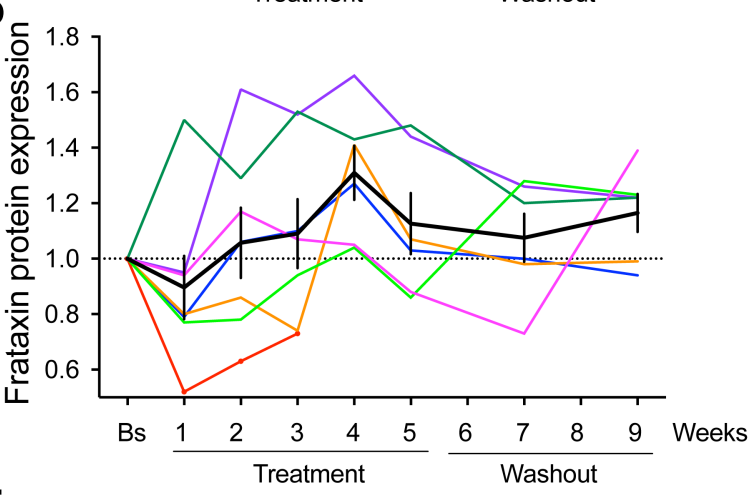

\section{F}

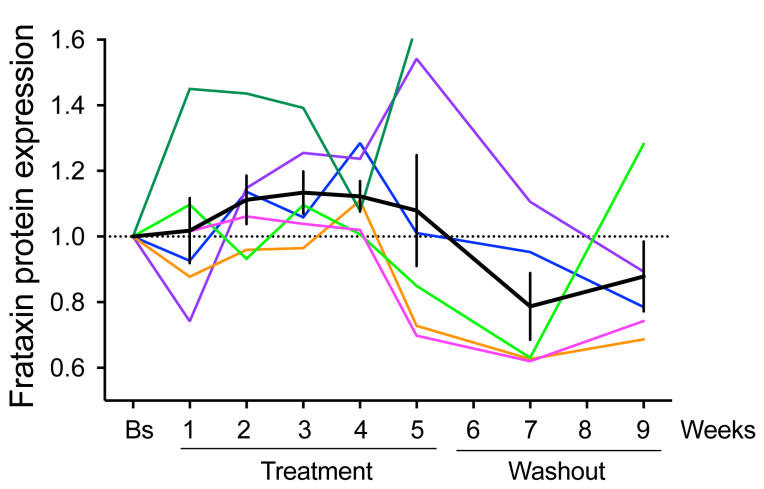

H

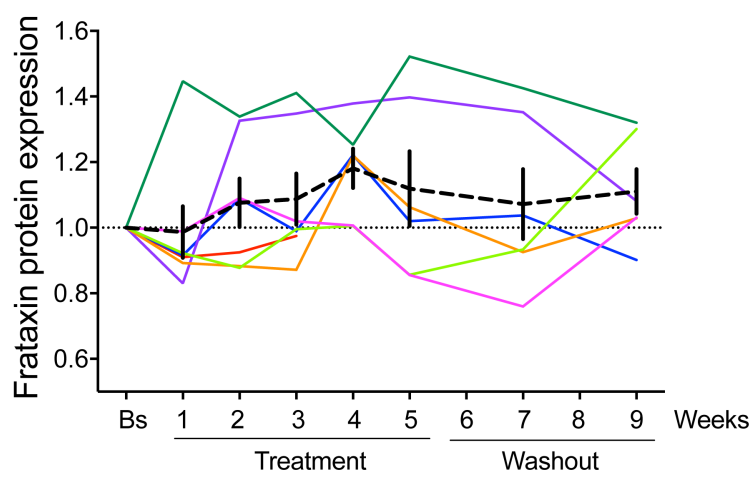

C

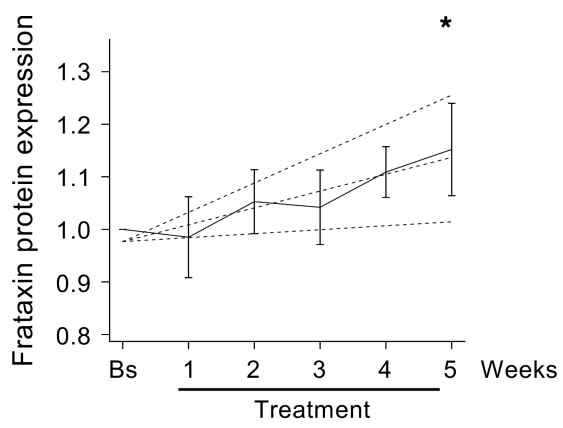

E

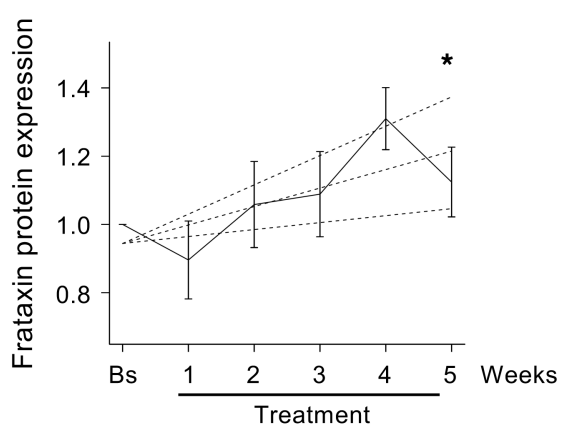

G

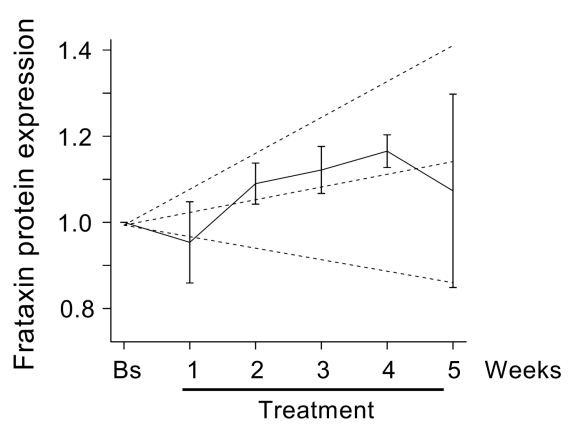

I

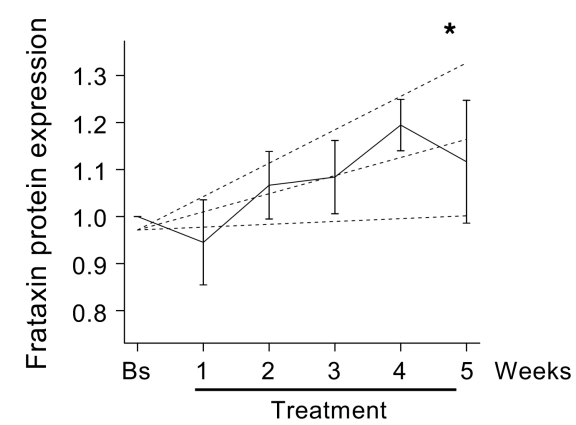


Figure 6. Exenatide induces frataxin protein in FRDA patients' platelets. (A) Schematic representation of the clinical trial. BID, twice daily. (B, D, F, H) Spaghetti plots of the changes in frataxin expression relative to baseline (Bs) in platelets over 5-week exenatide treatment followed by 4-week washout. Frataxin protein was measured by dipstick (B and $\mathbf{C})$, Western blot ( $\mathbf{D}$ and $\mathbf{E})$, and ELISA (F and $\mathbf{G})$. Panels $\mathbf{H}$ and I represent average frataxin expression measured by Western blot, dipstick, and ELISA. Colored lines represent individual patients $(n=7)$, with the smaller GAA repeat length in FXN shown for each at the top of panel B. The thick black lines indicate mean \pm SEM for each time point. (C, E, G, I) Statistical analysis: dashed lines indicate nonparametric trend line fitted to the mean for each data point (solid black line) and $95 \%$ confidence intervals generated by bootstrapping $(n=1000)$. ${ }^{*} P<0.05$ for a sustained increase in frataxin protein expression calculated by Fisher's transformation.

showed that 5-week exenatide treatment induces frataxin expression in patients' platelets. We propose that 2 main mechanisms of action underlie the putative beneficial effects of GLP-1 analogs in FRDA, namely improvement of oxidative stress and frataxin induction.

The insulinotropic effect of GLP-1 analogs relies on cAMP-mediated protein kinase A activation that increases insulin biosynthesis and secretion. GLP-1 analogs also have cytoprotective effects that may be beneficial for frataxin-deficient cells: they improve mitochondrial function, reduce expression of the proapoptotic thioredoxin-interacting protein Txnip, inhibit c-Jun N-terminal kinase, activate protein kinase A, and exchange protein activated by cAMP-dependent signaling, leading to enhanced expression of the antioxidant enzymes glutathione peroxidase, manganese superoxide dismutase, and catalase and thereby reducing oxidative stress (52-57). The metabolic studies in KIKO and WT mice showed that frataxin-deficient animals have greater responses to GLP-1 analogs, with clearer improvement in $\beta$ cell function (Figure 1 and Supplemental Figure 1). Frataxin-deficient mice might have a better response to GLP-1 analogs through alleviation of oxidative stress, which is present in patients with FRDA and frataxin-deficient $\beta$ cells and neurons and contributes to cellular dysfunction and death $(17,58,59)$. Prolonged oxidative stress reduces insulin gene expression and insulin content and causes $\beta$ cell dysfunction $(60,61)$. Our data indicate that high-fat feeding reduces insulin content, and this was partially restored by exenatide in KIKO mice (Supplemental Figure 1M). We have previously demonstrated that forskolin improves mitochondrial oxidative status in frataxin-deficient $\beta$ cells (17) and show here that exenatide alleviates oxidative stress in FRDA patients' iPSC-derived sensory neurons (Figure 5).

Table 1. Baseline characteristics of patients with FRDA

\begin{tabular}{|c|c|c|c|c|c|c|c|c|c|c|c|c|c|c|}
\hline & Sex & $\begin{array}{c}\text { Age } \\
\text { (years) }\end{array}$ & GAA & GAA & $\begin{array}{c}\text { Age at } \\
\text { onset } \\
\text { (years) }\end{array}$ & $\begin{array}{c}\text { Age at } \\
\text { diagnosis } \\
\text { (years) }\end{array}$ & $\begin{array}{l}\text { Disease } \\
\text { duration } \\
\text { (years) }\end{array}$ & $\begin{array}{c}\text { Systolic } \\
\text { BP } \\
(\mathrm{mm})\end{array}$ & $\begin{array}{c}\text { Diastolic } \\
\text { BP } \\
(\mathrm{mm})\end{array}$ & $\begin{array}{l}\text { Pulse } \\
\text { (bpm) }\end{array}$ & $\begin{array}{c}\text { Chol } \\
\text { (mg/dL) }\end{array}$ & $\begin{array}{c}\text { HDL } \\
\text { (mg/dL) }\end{array}$ & $\begin{array}{c}\text { LDL } \\
\text { (mg/dL) }\end{array}$ & $\begin{array}{l}\text { Triglycerides } \\
\text { (mg/dL) }\end{array}$ \\
\hline ID-01 & $\mathrm{F}$ & 19 & 845 & 980 & 8 & 12 & 11 & 110 & 67 & 108 & 160 & 40 & 106 & 70 \\
\hline ID-03 & $\mathrm{F}$ & 45 & 480 & 650 & 20 & 24 & 21 & 140 & 74 & 86 & 204 & 48 & 136 & 100 \\
\hline ID-04 & $\mathrm{F}$ & 50 & 850 & 850 & 10 & 14 & 40 & 109 & 66 & 70 & 147 & 47 & 77 & 113 \\
\hline ID-07 & $\mathrm{F}$ & 29 & 612 & 780 & 15 & 20 & 9 & 110 & 67 & 67 & 139 & 58 & 71 & 51 \\
\hline ID-08 & M & 32 & 412 & 545 & 19 & 24 & 8 & 110 & 64 & 78 & 158 & 63 & 87 & 41 \\
\hline ID-09 & $\mathrm{F}$ & 30 & 750 & 912 & 16 & 22 & 8 & 106 & 70 & 81 & 152 & 46 & 93 & 65 \\
\hline ID-11 & M & 23 & 700 & 980 & 14 & 16 & 7 & 123 & 80 & 71 & 154 & 39 & 92 & 113 \\
\hline ID-12 & $M$ & 21 & 816 & 1016 & 14 & 18 & 3 & 121 & 77 & 62 & 172 & 47 & 112 & 64 \\
\hline ID-17 & $M$ & 33 & 580 & 720 & 11 & 13 & 20 & 137 & 82 & 72 & 220 & 65 & 135 & 99 \\
\hline $\begin{array}{l}\text { Mean } \\
\pm \text { SEM }\end{array}$ & & $32 \pm 3$ & $654 \pm 56$ & $869 \pm 43$ & $14 \pm 1$ & $18 \pm 2$ & $15 \pm 3$ & $123 \pm 3$ & $77 \pm 3$ & $82 \pm 5$ & $172 \pm 9$ & $50 \pm 2$ & $101 \pm 7$ & $99 \pm 14$ \\
\hline
\end{tabular}

Participants 01-09 were in the liraglutide arm and participants 11-17 in the exenatide arm. BP, blood pressure; Chol, cholesterol; F, female; GAA, GAA repeat expansion number of the smaller (first column) and larger (second column) FXN alleles; M, male. 
Table 2. Adverse events reported during the 5-week liraglutide or exenatide treatment

\begin{tabular}{|c|c|c|c|c|c|c|c|c|c|c|c|c|}
\hline \multirow[t]{2}{*}{ Adverse event } & \multicolumn{6}{|c|}{ Liraglutide $(n=9)$} & \multicolumn{6}{|c|}{ Exenatide $(n=7)$} \\
\hline & W1 & W2 & W3 & W4 & W5 & $\%$ & W1 & W2 & W3 & W4 & W5 & $\%$ \\
\hline Nausea & 4 & 4 & 4 & 6 & 4 & 78 & 5 & 5 & 4 & 3 & 2 & 86 \\
\hline Constipation & 2 & 2 & 2 & 2 & 2 & 44 & 0 & 0 & 0 & 0 & 0 & 0 \\
\hline Dysgeusia & 1 & 2 & 2 & 2 & 2 & 22 & 0 & 0 & 0 & 0 & 0 & 0 \\
\hline Epigastric pain & 3 & 3 & 3 & 3 & 2 & 44 & 0 & 0 & 0 & 0 & 0 & 0 \\
\hline Patient dropout & 0 & 0 & 1 & 0 & 0 & 11 & 0 & 0 & 0 & 1 & 0 & 14 \\
\hline
\end{tabular}

Data show the number of patients in the treatment arm experiencing a side effect at the indicated week (W); \% shows the percentage of patients reporting the adverse event at least once during treatment. The reason for dropout in the liraglutide arm was a nonserious adverse event; the reason for dropout in the exenatide arm was not related to an adverse event.

A beneficial impact of GLP-1 analogs was reported in a model of Wolfram syndrome 2, an autosomal recessive disease caused by mitochondrial membrane protein nutrient-deprivation autophagy factor-1 (NAF1, also known as CISD2) deficiency (62). NAF1 is involved in ISC transfer into apoproteins. Its deficiency in $\beta$ cells leads to mitochondrial iron accumulation, mitochondrial dysfunction, oxidative stress, and reduced insulin secretion and content, defects that were partially corrected by exenatide (62). Mitochondrial iron accumulation is a hallmark of FRDA (63), but whether GLP-1 analogs improve this remains to be determined. GLP-1 analogs increase $\beta$ cell antioxidant capacity by inducing NF-E2-related factor (NRF2), a master regulator of redox homeostasis, and its target, glutathione reductase (56). NRF2 signaling is defective in frataxin deficiency models and in patients with FRDA (64-66), and NRF2 inducers have been proposed as neuroprotective drugs for FRDA (67). GLP-1 analog-mediated NRF2 induction may be beneficial for frataxin-deficient $\beta$ cells and neurons.

The present data also show that cAMP induction by exenatide, forskolin, and GIP induces frataxin protein expression in rat, mouse, and human cells without affecting frataxin mRNA (Figure 2, Figure 3 , Figure 4, Figure 5, Figure 6, Supplemental Figure 3, Supplemental Figure 6, and Supplemental Figure 7). Exenatide has been shown to enhance selected protein synthesis in mouse pancreas without modifying gene transcription (68); the mechanism(s) remains to be elucidated. The frataxin induction by cAMP inducers, even if modest, may be sufficient to beneficially affect FRDA pathophysiology and hence be clinically relevant. Indeed, in patients with FRDA, higher frataxin protein levels associate with less severe phenotype, and heterozygous carriers of an FXN GAA repeat expansion, who have half of normal frataxin levels (range 30\%-80\%), remain asymptomatic (69). In in vitro and in vivo rodent models, GLP-1 analogs induced expression of frataxin and its downstream targets. The same effects were seen in FRDA patient iPSC-derived sensory neurons, together with improved mitochondrial function. Exenatide administration to patients with FRDA led to modest platelet frataxin protein induction (Figure 6). The response in this pilot study was heterogeneous, with a hint to better responses occurring in patients with larger GAA1 repeat expansions (i.e., less frataxin expression and more severe disease). The study was neither designed nor powered to assess this, but future incretin analog trials could evaluate whether GAA repeat size predicts therapeutic response, and this might guide patient stratification. Some of the variability in the data may be related to the sensitivity of frataxin protein assay methods. The study was not designed to assess beneficial effects of GLP-1 analogs on neurologic manifestations of FRDA because it was very short and did not include a placebo group. Besides the well-known gastrointestinal side effects, there were no adverse events, suggesting that these molecules can safely be tested in larger trials.

The choice of an incretin analog to treat FRDA should take into consideration the drug's crossing of the blood-brain barrier. Several reports describe that exenatide and GLP-1 analogs with higher molecular weight (through linkage with fatty acids and/or carrier proteins) can directly access the brain, especially the hypothalamus $(70,71)$. The magnitude seems, however, limited from liraglutide levels measured in cerebrospinal fluid (72). More data are needed to assess this for different GLP-1 analogs. Through inhibitory effects on inflammation and apoptosis, GLP-1 and/or GIP analogs have been shown to confer 


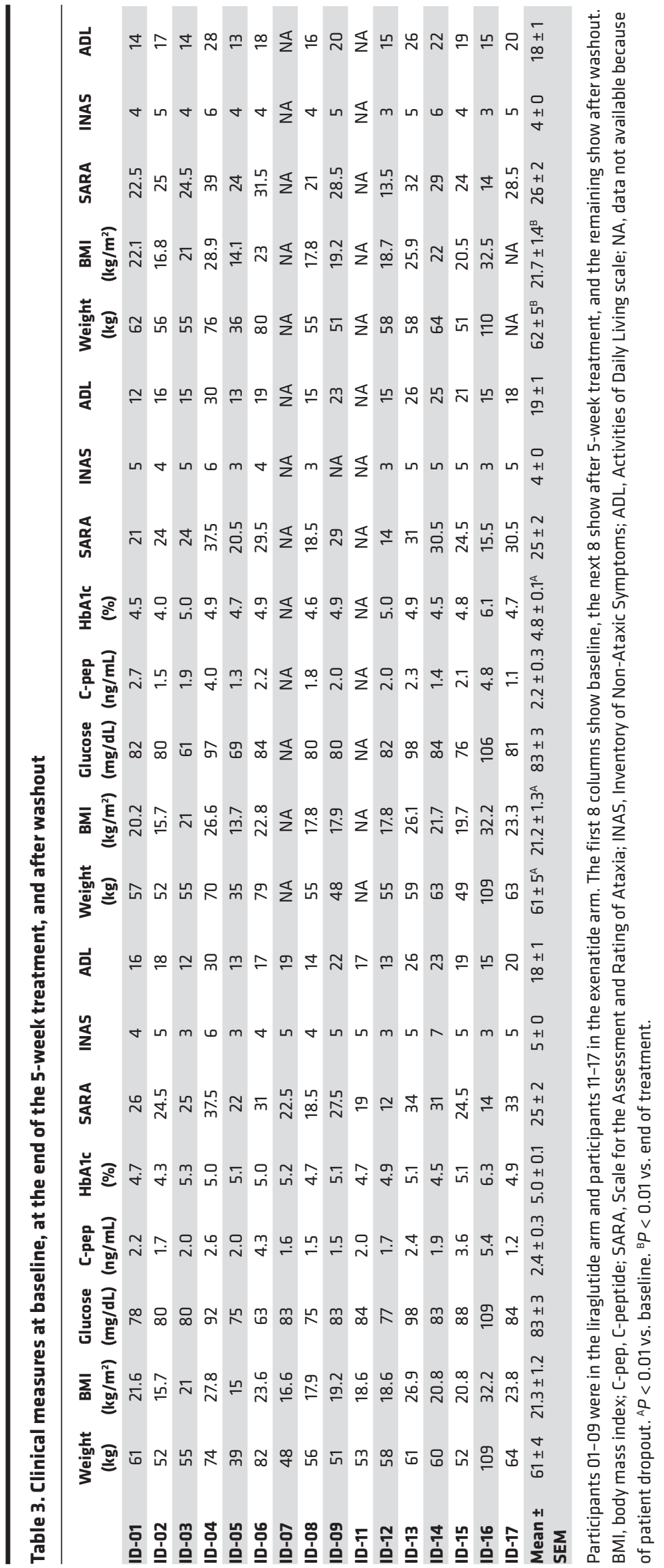

neuroprotection in traumatic and neurodegenerative Parkinson's and Alzheimer's disease $(73,74)$.

Altogether, our preclinical and clinical data indicate that GLP-1 analogs are beneficial for frataxin-deficient $\beta$ cells and neurons. They improve $\beta$ cell function, reduce oxidative stress, and improve mitochondrial function of FRDA patient sensory neurons, possibly, but not exclusively, through a frataxin-inducing effect. These data provide a strong rationale for the design of a long-term clinical trial to assess the disease-modifying effect of GLP-1 analogs in patients with FRDA.

\section{Methods}

\section{Dietary intervention and exenatide treatment in mice}

Male frataxin-deficient KIKO (stock number 014162, The Jackson Laboratory) and WT C57BL/6J mice were fed a regular $(10 \% \mathrm{kcal}$ fat, Research Diets D12450B) or high-fat diet (60\% kcal fat, Research Diets D12492) at the age of $12-14$ weeks (18-23 animals per group). After 15 weeks, mice were randomized to exenatide or vehicle. Mini-osmotic Alzet 2006 pumps (Durect) were subcutaneously implanted in the interscapular area to administer exenatide $(10 \mu \mathrm{g} / \mathrm{kg} /$ day, AstraZeneca) or vehicle (saline with $0.1 \%$ mouse albumin). Pumps were replaced after 6 weeks for a total treatment period of 12 weeks. Metabolic tests were performed at the end of the 27-week dietary intervention. Mice were killed by cervical dislocation and heart, cerebrum, cerebellum, spine, and islets were isolated.

\section{Metabolic studies in mice}

IPGTTs and ITTs were done as described previously (75). For the IPGTT, after a 16-hour fast, $2 \mathrm{mg}$ glucose/g body weight was administered and glycemia measured (glucometer Accu-Chek Aviva Nano, Roche) before and after 15, 30, 60, 90, and 120 minutes. Blood was collected from the tail vein and plasma insulin measured using ultrasensitive mouse insulin ELISA (Crystal Chem). Glucose tolerance was calculated as the AUC from 0 to 120 minutes. For the ITT, 4-hour-fasted mice were given insulin IP (0.75 $\mathrm{mU}$ Actrapid/g weight, Novo Nordisk), and glycemia was measured before and after 15, 30, 60, 90, and 120 minutes. Insulin sensitivity was calculated as the AOC from 0 to 30 minutes in the ITT and by HOMA-IR ([fasting glucose $\mathrm{mg} / \mathrm{dL} \times$ fasting insulin $\mu \mathrm{U} / \mathrm{mL}] /$ 405). Insulinogenic index was calculated as $\Delta \mathrm{I}$ over $\Delta \mathrm{G}$ in the first 15 minutes of IPGTT. Function of $\beta$ cells was calculated as insulinogenic index $\times$ insulin sensitivity measure ITT AOC. 
Mouse islet isolation and ex vivo glucose-induced insulin secretion

Islets were isolated by injecting Medium 199 (Gibco, Thermo Fisher Scientific) containing $1 \mathrm{mg} / \mathrm{mL}$ collagenase from Clostridium histolyticum (MilliporeSigma) into the bile duct. Dissected pancreas was incubated for 17 minutes at $37^{\circ} \mathrm{C}$ to ensure digestion. After 3 washes with Medium 199 containing 10\% FBS, islets were separated from exocrine tissue by density gradient Histopaque-1077 (MilliporeSigma). Islets were washed twice and handpicked for insulin secretion studies. After 30 minutes' incubation in Medium 199 containing $100 \mathrm{mg} / \mathrm{dL}$ glucose and 10\% FBS, islets were washed with Krebs-Ringer bicarbonate HEPES solution, and insulin secretion was induced by sequential 1-hour incubations in Krebs-Ringer bicarbonate HEPES containing 30 or $300 \mathrm{mg} / \mathrm{dL}$ glucose. Insulin was measured by ultrasensitive mouse insulin ELISA and total protein by Bradford assay (Bio-Rad).

\section{Neuronal size in dorsal root ganglia}

Mouse lumbar spine sections were fixed in $4 \%$ formalin for 72 hours, washed under running water, and immersed for 48 hours in decalcifying solution consisting of equal parts of $8 \%$ hydrochloric acid and $8 \%$ formic acid (refreshed after 24 hours). Samples were rinsed in water, immersed in neutralizing ammonia solution for 30 minutes, and washed under running water for 24 hours. Cassettes were immersed in $70 \%$ isopropanol and stored at $4^{\circ} \mathrm{C}$ until paraffin embedding. Sequential sections, $6 \mu \mathrm{m}$ thick, were stained with hematoxylin and eosin (MilliporeSigma), and digital images of cross-sectional areas of dorsal root ganglia were obtained. The number and area of 50-450 dorsal root ganglia neuronal cells per mouse from 4-5 mice per group were quantified by 2 blinded users with ImageJ.

\section{iPSC culture and differentiation into $\beta$ cells}

Skin fibroblasts from a 26-year-old male patient with FRDA were reprogrammed into iPSCs using 3 episomal vectors with oriP/EBNA-1 backbone containing reprogramming factors OCT3/4, SOX2, KLF4, L-MYC, LIN28, and p53 shRNA. iPSCs were cultured in Matrigel-coated plates (Corning BV, Life Sciences) in E8 medium (Life Technologies) and passaged with $0.5 \mathrm{mM}$ EDTA (Life Technologies) twice weekly. iPSCs were differentiated into $\beta$ cells as described (47) with slight modifications. Briefly, iPSCs were plated at high density $\left(3 \times 10^{6}\right.$ cells/well) in E8 medium containing $5 \mu \mathrm{M}$ ROCK inhibitor (STEMCELL Technologies). Differentiation was initiated at confluence (after 24-48 hours). Until pancreatic progenitor stage (stage 4), cells were differentiated in Matrigel-coated wells, after which they were detached and plated into $400-\mu \mathrm{m}$ diameter microwell plates at 750 cells/microwell (AggreWell, STEMCELL Technologies) to form islet-like aggregates. The differentiation was continued in microwells. Between days 0 and 15 of differentiation, medium was freshly prepared and changed daily; from day 16 until the end of differentiation, medium was refreshed every second day.

Human islets and EndoC- $\beta \mathrm{H} 1$ cells

Human islets from nondiabetic organ donors ( $n=5$, age $63 \pm 7$ years, body mass index $27 \pm 2 \mathrm{~kg} / \mathrm{m}^{2}$, causes of death 2 cerebral hemorrhage, postanoxic encephalopathy, cardiovascular disease, and trauma) were isolated by collagenase digestion and density gradient purification (76). In these islets the percentage of $\beta$ cells, assessed by insulin immunofluorescence, was $43 \% \pm 7 \%$. Human EndoC- $\beta \mathrm{H} 1$ cells (77), provided by R. Scharfmann (Cochin Institute, Paris, France), were cultured as described (78). Cells were tested monthly to rule out mycoplasma contamination.

\section{iPSC differentiation into neurons and sensory neurons}

Two previously characterized FRDA iPSC lines (FA135, FA141, 2 clones of each) (43), 1 newly reprogrammed FRDA iPSC line (HEL135.2), and 2 previously characterized control iPSC lines (HEL46.11 and HEL115.6) $(47,48)$ were used for neuronal differentiation, as previously described $(17,43)$. For sensory neuron differentiation (46), iPSCs were cultured to $60 \%-70 \%$ confluence, dissociated with accutase for 5 minutes, pelleted at $250 \mathrm{~g}$ for 3 minutes, plated on Matrigel-coated plates at 26,000 cells/ $\mathrm{cm}^{2}$, and cultured in E8 medium with $5 \mu \mathrm{M}$ ROCK inhibitor for 24 hours. Differentiation medium DMEM-F12 containing 10\% KnockOut Serum Replacement (Thermo Fisher Scientific), $0.3 \mu$ M LDN193189 (Cellagen Technology), $2 \mu$ M A83-01 (Cellagen Technology), $6 \mu$ M CHIR99021 (STEMCELL Technologies), $2 \mu \mathrm{M}$ RO4929097 (Cellagen Technology), $3 \mu \mathrm{M}$ SU5402 (Tocris), and $0.3 \mu \mathrm{M}$ retinoic acid (MilliporeSigma) (46) was changed every second day. After 8 days most cells reached the sensory neuron state. Exenatide was added to neurobasal maintenance medium supplemented with $10 \mathrm{ng} / \mathrm{mL}$ 
neurotrophin 3 (PeproTech), $20 \mathrm{ng} / \mathrm{mL}$ brain-derived neurotrophic factor (PeproTech), $20 \mathrm{ng} / \mathrm{mL}$ nerve growth factor (PeproTech), and $20 \mathrm{ng} / \mathrm{mL}$ glial cell-derived growth factor (Bio-Techne) (46).

\section{Protein extraction}

Total protein was extracted from mouse tissues and iPSC-neurons in RIPA buffer (MilliporeSigma) containing protease inhibitors (cOmplete, EDTA-free protease inhibitor cocktail, Roche). Tissues were homogenized using T10 basic Ultra-Turrax disperser (IKA). Mouse islets and iPSC-neurons were sonicated in Bioruptor NGS (Diagenode). Total extracts were clarified by centrifugation (14,000 $g$ for 10 minutes at $4^{\circ} \mathrm{C}$ ), and protein was quantified by BCA method (Thermo Fisher Scientific).

\section{Pilot clinical trial}

Study design. The study was a small, single-center, open-label, active-comparator, 2-armed, parallel-group intervention trial using liraglutide (Victoza, Novo Nordisk) or exenatide (Byetta, AstraZeneca) in patients with FRDA. The study was conducted at the Erasmus Hospital and had an 11-week duration: 2 weeks for screening visit, obtaining informed consent, collecting 2 baseline blood samples to measure frataxin expression, and educating on drug self-administration; 5-week GLP-1 analog treatment; and 4-week washout. The primary objective was to assess the frataxin-inducing effect of exenatide or liraglutide in patient blood samples, collected weekly during treatment and after 2 and 4 weeks of washout, and to evaluate safety and tolerability. Frataxin protein and mRNA expression before, during, and after treatment was assessed in PBMCs and platelets by lateral flow dipstick immunoassay, ELISA, Western blot, and real-time PCR. Drug safety and tolerability were assessed by weekly visits and hematology and chemistry tests at the start and end of treatment. The secondary objective was to evaluate changes in FRDA clinical rating scales, using the Scale for the Assessment and Rating of Ataxia, Inventory of Non-Ataxic Symptoms, and Activities of Daily Living scales, before and at the end of treatment and after washout.

Recruitment. Patients were contacted via neurologists in reference centers and through the European Friedreich's Ataxia Consortium for Translational Studies and Friedreich's Ataxia Research Alliance patient consortia in Europe. Sixteen participants were enrolled, 9 in the liraglutide group and 7 in the exenatide group. Participant characteristics are summarized in Table 1.

Inclusion criteria. Male or female 18- to 70-year-old patients with FRDA diagnosis confirmed by genetic testing, receiving stable doses of medications for 30 days prior to enrollment and for the duration of the study, were included. Patients with insulin-treated diabetes, a history of acute or chronic pancreatitis, gastric or other major bleeding, thyroid neoplasia, hypothyroidism, or hyperthyroidism were excluded, as were patients who had previously experienced side effects from GLP-1 analogs or who had received another investigational product in the past 28 days.

Treatment. Participants were assigned to liraglutide or exenatide. There was no formal randomization process. During the first week, the liraglutide group received a starting dose of $0.6 \mathrm{mg}$ liraglutide once daily. This dose was increased to $1.2 \mathrm{mg} / \mathrm{kg}$ during the following 2 weeks and $1.8 \mathrm{mg} / \mathrm{kg}$ for the last 2 treatment weeks. The exenatide group received $5 \mu \mathrm{g}$ exenatide twice daily during the first week of treatment and the standard dose of $10 \mu \mathrm{g}$ twice daily for the following 4 weeks.

PBMC and platelet sample preparation. Blood from patients with FRDA and 2 control individuals was collected in sodium citrate tubes (BD Biosciences), and PBMCs and platelets were purified by differential centrifugation and Ficoll gradient. For protein extraction, cells were lysed by sonication in ice-cold MitoSciences extraction buffer (Abcam) with protease inhibitors (cOmplete, Roche). Cell extracts were cleared by centrifugation at $14,000 \mathrm{~g}$ for 20 minutes at $4^{\circ} \mathrm{C}$. Supernatant was stored at $-80^{\circ} \mathrm{C}$ until frataxin protein quantification.

\section{Frataxin protein quantification}

MitoSciences dipstick assay (Abcam) was used to measure frataxin protein in PBMCs and platelets. We used $10 \mu \mathrm{g}$ total protein from PBMCs and $15 \mu \mathrm{g}$ from platelets; dipsticks were quantified with Hamamatsu immunochromato MS1000 Dipstick reader.

Human and mouse frataxin ELISAs (Abcam) were performed according to the manufacturer's instructions. Samples were diluted in lysis buffer provided with the kit and equal sample volumes used in the assay. Frataxin protein was normalized to total protein.

For Western blotting, 15-20 $\mu$ g total protein was loaded on $14 \%$ SDS-polyacrylamide or $4 \%-15 \%$ precast Mini PROTEAN polyacrylamide gels (Bio-Rad). Proteins were transferred to nitrocellulose membranes, 
blocked with $5 \%$ nonfat milk, and incubated with antibodies against frataxin, GAPDH, $\beta$-actin, COX IV, aconitase, NDUFS3, mitochondrial superoxide dismutase, and lipoic acid (to detect lipoic acid-containing PDH and $\mathrm{OGDH}$ ) (Supplemental Table 1). After incubation with secondary horseradish peroxidase-conjugated antibodies, proteins were detected using SuperSignal West Femto chemiluminescence revealing reagent (Thermo Fisher Scientific) in ChemiDoc XRS+ system and quantified by Image Lab software (Bio-Rad).

\section{Immunofluorescence}

Cells were fixed in 4\% formaldehyde for 15-20 minutes, permeabilized with $0.5 \%$ Triton X-100 for 10 minutes, blocked with UltraV block (Thermo Fisher Scientific) for 8-10 minutes, and incubated with primary antibodies (Supplemental Table 1) diluted in $0.1 \%$ Tween in PBS overnight at $4^{\circ} \mathrm{C}$. Following incubation with secondary antibodies for 30 minutes at room temperature, samples were mounted with Vectashield with DAPI (Vector Laboratories) and covered with glass coverslips.

\section{Detection of intracellular reactive oxygen species}

Reactive oxygen species were measured with the oxidation-sensitive fluorescent probe HPF (Thermo Fisher Scientific) in cells cultured in black-bottom plates. Cells were incubated with $10 \mu \mathrm{M}$ HPF for 30 minutes and washed twice with PBS, and fluorescence was measured in VICTOR multilabel plate reader (PerkinElmer) using excitation and emission spectra of 485 and $535 \mathrm{~nm}$.

\section{Mitochondrial respiration}

OCRs of sensory neurons were measured using XFp Extracellular Flux Analyzer (Seahorse Bioscience). Cells were preincubated in Seahorse medium at $\mathrm{pH} 7.4$ containing $7 \mathrm{mg} / \mathrm{mL}$ glucose and $2 \mathrm{mM}$ Glutamax for 1 hour at $37^{\circ} \mathrm{C}$ in a non- $\mathrm{CO}_{2}$ incubator. Mitochondrial respiration was measured basally and after injection of $1.5 \mu \mathrm{M}$ oligomycin, $2 \mu \mathrm{M}$ FCCP, and $1 \mu \mathrm{M}$ rotenone plus $1 \mu \mathrm{M}$ antimycin A. Data were normalized to total protein. Basal respiration was calculated by subtracting nonmitochondrial respiration from the last measurement before oligomycin injection, ATP production by subtracting the minimum measurement after oligomycin injection from the last baseline measurement, and maximal respiration by subtracting nonmitochondrial respiration from maximum measurement after FCCP injection.

\section{mRNA extraction and real-time PCR}

Total RNA from tissues and cells was extracted using RNeasy Plus Micro Kit (Qiagen). mRNA was reverse-transcribed as described (17) and assessed by real-time PCR using Rotor-Gene SYBR Green on Rotor-Gene Q cycler (Qiagen) or Q SYBR Green Supermix (Bio-Rad) on a MyiQ2 instrument (single color, Bio-Rad). Gene expression was calculated as copies/ $\mu \mathrm{L}$ using the standard curve approach. Standards were prepared in conventional PCR. Expression was corrected for the geometric mean of reference genes GAPDH and $\beta$-actin. Primer sequences are described in Supplemental Tables 2 and 3.

Insulin secretion and NAD(P)H autofluorescence in iPSC-derived $\beta$ cells

For insulin secretion, 40 iPSC- $\beta$ cell aggregates per condition were washed in glucose-free Krebs buffer (Univercell-Biosolutions), preincubated 30 minutes in $30 \mathrm{mg} / \mathrm{dL}$ glucose, and then incubated at low (30 mg/dL) or high glucose $(300 \mathrm{mg} / \mathrm{dL})$ and high glucose plus forskolin $(10 \mu \mathrm{M})$ for 1 hour. Insulin released to supernatant and insulin content were analyzed by human insulin ELISA (Mercodia) and normalized to total protein (BioRad protein assay). For NAD(P)H measurements, aggregates were incubated for 2 hours in glucose-free, bicarbonate-buffered Krebs and then perifused (flow rate $\sim 1 \mathrm{~mL} / \mathrm{min}$ ) with Krebs containing 0-360 mg/dL glucose. $\mathrm{NAD}(\mathrm{P}) \mathrm{H}$ autofluorescence was recorded by microspectrofluorometry in 6 perifused aggregates as described(79). Data were normalized to fluorescence levels measured 15 minutes after addition of $10 \mu \mathrm{M}$ FCCP.

\section{Statistics}

Data are shown using box plots, with the median represented by horizontal line and 25th and 75th percentiles at the bottom and top of the box. Comparisons between groups were performed by Kruskal-Wallis test followed by Benjamini, Krieger, and Yekutieli correction for multiple comparisons, unpaired 2-tailed $t$ test followed by Holm-Šídák correction for multiple comparisons, or 1- or 2-way ANOVA followed by unpaired or paired 2-tailed $t$ test with Bonferroni's or Tukey's correction for multiple comparisons. $P<0.05$ or $q<0.05$ was considered statistically significant. 


\section{Study approval}

Animal experiments were approved by the Commission d'Ethique du Bien-Être Animal of the ULB (reference $647 \mathrm{~N}$ ) and are in accordance with the Guide for the Care and Use of Laboratory Animals (National Academies Press, 2011) from the Institute for Laboratory Animal Research, National Research Council.

Human pancreata not suitable for clinical purposes were collected from nondiabetic, brain-dead organ donors after obtaining written informed consent from next of kin and handled as described (80) with the approval of the Ethical Committee, University of Pisa, in Pisa.

FRDA patient skin fibroblasts were obtained after written informed consent was given by patients with approval by the Erasmus Hospital Ethics Committee.

The clinical trial with GLP-1 analogs in patients with FRDA was approved by the Erasmus Hospital Ethics Committee (reference P2014/311) and submitted to the European Clinical Trials Database (EudraCT/CCB 2014-003598-41). Participants provided written informed consent prior to inclusion.

\section{Author contributions}

MIE, MB, MP, DLE, and MC conceived the study; AFO, CC, FF, ST, SC, and JCJ developed the methodology; ML performed formal analysis; MIE, AFO, CC, FF, CD, ST, AH, SC, NP, YC, BA, MT, MB, and MC investigated; MIE and MC wrote the original draft; FF, CD, YC, PM, JCJ, MP, and DLE reviewed and edited the manuscript; MIE, MP, DLE, and MC acquired funding; MR, LM, and PM provided resources; and MC supervised.

\section{Acknowledgments}

This work was supported by the Fonds Erasme Olivia De Clercq, Belgium; the Phillip Bennett and Kyle Bryant Translational Research Award of the Friedreich Ataxia Research Alliance, USA; the European Union's Horizon 2020 research and innovation programme, project T2DSystems, under grant agreement 667191; the Brussels Region Innoviris project DiaType; and the Fonds National de la Recherche Scientifique (FNRS), Belgium. AFO and BA were Fund for Research Training in Industry and AgricultureFNRS (FRIA-FNRS) fellows.

We thank Isabelle Millard, Michael Pangerl, and Anyishai Musuaya from the ULB Center for Diabetes Research for excellent technical support and Nick Alaerts from the Neurology Department of the Erasmus Hospital for invaluable help with the clinical trial.

Address correspondence to: Mariana Igoillo-Esteve or Miriam Cnop, ULB Center for Diabetes Research, Université Libre de Bruxelles CP-618, Route de Lennik 808, 1070 Brussels, Belgium. Phone: 32.2.555.63.05; Email: migoillo@ulb.ac.be (MIE); mcnop@ulb.ac.be (MC).

SC's present address is: Translational Molecular Neuroscience Group, Weatherall Institute of Molecular Medicine, Nuffield Department of Clinical Neurosciences, University of Oxford, Oxford, United Kingdom.

1. Campuzano V, et al. Friedreich's ataxia: autosomal recessive disease caused by an intronic GAA triplet repeat expansion. Science. 1996;271(5254):1423-1427.

2. Saveliev A, Everett C, Sharpe T, Webster Z, Festenstein R. DNA triplet repeats mediate heterochromatin-protein-1-sensitive variegated gene silencing. Nature. 2003;422(6934):909-913.

3. Silva AM, Brown JM, Buckle VJ, Wade-Martins R, Lufino MM. Expanded GAA repeats impair FXN gene expression and reposition the FXN locus to the nuclear lamina in single cells. Hum Mol Genet. 2015;24(12):3457-3471.

4. Herman D, Jenssen K, Burnett R, Soragni E, Perlman SL, Gottesfeld JM. Histone deacetylase inhibitors reverse gene silencing in Friedreich's ataxia. Nat Chem Biol. 2006;2(10):551-558.

5. Al-Mahdawi S, et al. The Friedreich ataxia GAA repeat expansion mutation induces comparable epigenetic changes in human and transgenic mouse brain and heart tissues. Hum Mol Genet. 2008;17(5):735-746.

6. Koeppen AH. Friedreich's ataxia: pathology, pathogenesis, and molecular genetics. J Neurol Sci. 2011;303(1-2):1-12.

7. Rötig A, et al. Aconitase and mitochondrial iron-sulphur protein deficiency in Friedreich ataxia. Nat Genet. 1997;17(2):215-217.

8. Babcock M, et al. Regulation of mitochondrial iron accumulation by Yfh1p, a putative homolog of frataxin. Science. 1997;276(5319):1709-1712.

9. Pandolfo M. Friedreich ataxia: the clinical picture. J Neurol. 2009;256 Supp1 1:3-8.

10. Harding AE. Friedreich's ataxia: a clinical and genetic study of 90 families with an analysis of early diagnostic criteria and 
intrafamilial clustering of clinical features. Brain. 1981;104(3):589-620.

11. Schoenle EJ, Boltshauser EJ, Baekkeskov S, Landin Olsson M, Torresani T, von Felten A. Preclinical and manifest diabetes mellitus in young patients with Friedreich's ataxia: no evidence of immune process behind the islet cell destruction. Diabetologia. 1989;32(6):378-381.

12. Finocchiaro G, Baio G, Micossi P, Pozza G, di Donato S. Glucose metabolism alterations in Friedreich's ataxia. Neurology. 1988;38(8):1292-1296.

13. Cnop M, et al. Central role and mechanisms of $\beta$-cell dysfunction and death in Friedreich ataxia-associated diabetes. Ann Neurol. 2012;72(6):971-982.

14. Pousset F, et al. A 22-year follow-up study of long-term cardiac outcome and predictors of survival in Friedreich Ataxia. JAMA Neurol. 2015;72(11):1334-1341.

15. Koeppen AH, et al. The dentate nucleus in Friedreich's ataxia: the role of iron-responsive proteins. Acta Neuropathol. 2007;114(2):163-173.

16. Koeppen AH, Ramirez RL, Becker AB, Mazurkiewicz JE. Dorsal root ganglia in Friedreich ataxia: satellite cell proliferation and inflammation. Acta Neuropathol Commun. 2016;4(1):46.

17. Igoillo-Esteve M, et al. Unveiling a common mechanism of apoptosis in $\beta$-cells and neurons in Friedreich's ataxia. Hum Mol Genet. 2015;24(8):2274-2286.

18. Rustin P, von Kleist-Retzow JC, Chantrel-Groussard K, Sidi D, Munnich A, Rötig A. Effect of idebenone on cardiomyopathy in Friedreich's ataxia: a preliminary study. Lancet. 1999;354(9177):477-479.

19. Mariotti C, Solari A, Torta D, Marano L, Fiorentini C, Di Donato S. Idebenone treatment in Friedreich patients: one-year-long randomized placebo-controlled trial. Neurology. 2003;60(10):1676-1679.

20. Cooper JM, Korlipara LV, Hart PE, Bradley JL, Schapira AH. Coenzyme Q10 and vitamin E deficiency in Friedreich's ataxia: predictor of efficacy of vitamin E and coenzyme Q10 therapy. Eur J Neurol. 2008;15(12):1371-1379.

21. Kearney M, Orrell RW, Fahey M, Brassington R, Pandolfo M. Pharmacological treatments for Friedreich ataxia. Cochrane Database Syst Rev. 2016;(8):CD007791.

22. Tomassini B, et al. Interferon gamma upregulates frataxin and corrects the functional deficits in a Friedreich ataxia model. Hum Mol Genet. 2012;21(13):2855-2861.

23. Acquaviva F, et al. Recombinant human erythropoietin increases frataxin protein expression without increasing mRNA expression. Cerebellum. 2008;7(3):360-365.

24. Rai M, et al. HDAC inhibitors correct frataxin deficiency in a Friedreich ataxia mouse model. PLoS One. 2008;3(4):e1958.

25. Lynch DR, et al. Randomized, double-blind, placebo-controlled study of interferon- $\gamma$ 1b in Friedreich Ataxia. Ann Clin Transl Neurol. 2019;6(3):546-553.

26. Mariotti C, et al. Erythropoietin in Friedreich ataxia: no effect on frataxin in a randomized controlled trial. Mov Disord. 2012;27(3):446-449.

27. Soragni E, Gottesfeld JM. Translating HDAC inhibitors in Friedreich's ataxia. Expert Opin Orphan Drugs. 2016;4(9):961-970.

28. Drucker DJ. The biology of incretin hormones. Cell Metab. 2006;3(3):153-165.

29. Holst JJ. The physiology of glucagon-like peptide 1. Physiol Rev. 2007;87(4):1409-1439.

30. Vilsbøll T, Knop FK. Long-acting GLP-1 analogs for the treatment of type 2 diabetes mellitus. BioDrugs. 2008;22(4):251-257.

31. Finan B, et al. Unimolecular dual incretins maximize metabolic benefits in rodents, monkeys, and humans. Sci Transl Med. 2013;5(209):209ra151.

32. Campbell JE, Drucker DJ. Pharmacology, physiology, and mechanisms of incretin hormone action. Cell Metab. 2013;17(6):819-837.

33. Drucker DJ, Philippe J, Mojsov S, Chick WL, Habener JF. Glucagon-like peptide I stimulates insulin gene expression and increases cyclic AMP levels in a rat islet cell line. Proc Natl Acad Sci USA. 1987;84(10):3434-3438.

34. Widenmaier SB, et al. A GIP receptor agonist exhibits beta-cell anti-apoptotic actions in rat models of diabetes resulting in improved beta-cell function and glycemic control. PLoS One. 2010;5(3):e9590.

35. Yusta B, et al. GLP-1 receptor activation improves beta cell function and survival following induction of endoplasmic reticulum stress. Cell Metab. 2006;4(5):391-406.

36. Cunha DA, et al. Glucagon-like peptide-1 agonists protect pancreatic beta-cells from lipotoxic endoplasmic reticulum stress through upregulation of BiP and JunB. Diabetes. 2009;58(12):2851-2862.

37. Ban K, Noyan-Ashraf MH, Hoefer J, Bolz SS, Drucker DJ, Husain M. Cardioprotective and vasodilatory actions of glucagon-like peptide 1 receptor are mediated through both glucagon-like peptide 1 receptor-dependent and -independent pathways. Circulation. 2008;117(18):2340-2350.

38. Porter DW, Irwin N, Flatt PR, Hölscher C, Gault VA. Prolonged GIP receptor activation improves cognitive function, hippocampal synaptic plasticity and glucose homeostasis in high-fat fed mice. Eur J Pharmacol. 2011;650(2-3):688-693.

39. McClean PL, Parthsarathy V, Faivre E, Hölscher C. The diabetes drug liraglutide prevents degenerative processes in a mouse model of Alzheimer's disease. J Neurosci. 2011;31(17):6587-6594

40. Miranda CJ, et al. Frataxin knockin mouse. FEBS Lett. 2002;512(1-3):291-297.

41. Coppola G, et al. Gene expression profiling in frataxin deficient mice: microarray evidence for significant expression changes without detectable neurodegeneration. Neurobiol Dis. 2006;22(2):302-311.

42. Coppola G, et al. Functional genomic analysis of frataxin deficiency reveals tissue-specific alterations and identifies the PPAR gamma pathway as a therapeutic target in Friedreich's ataxia. Hum Mol Genet. 2009;18(13):2452-2461.

43. Hick A, et al. Neurons and cardiomyocytes derived from induced pluripotent stem cells as a model for mitochondrial defects in Friedreich's ataxia. Dis Model Mech. 2013;6(3):608-621.

44. Codazzi F, et al. Friedreich ataxia-induced pluripotent stem cell-derived neurons show a cellular phenotype that is corrected by a benzamide HDAC inhibitor. Hum Mol Genet. 2016;25(22):4847-4855.

45. Solmonson A, DeBerardinis RJ. Lipoic acid metabolism and mitochondrial redox regulation. J Biol Chem. 2018;293(20):7522-7530.

46. Cai S, Han L, Ao Q, Chan YS, Shum DK. Human induced pluripotent cell-derived sensory neurons for fate commitment of bone marrow-derived Schwann cells: implications for remyelination therapy. Stem Cells Transl Med. 2017;6(2):369-381

47. Cosentino C, et al. Pancreatic $\beta$-cell tRNA hypomethylation and fragmentation link TRMT10A deficiency with diabetes. Nucleic 
Acids Res. 2018;46(19):10302-10318.

48. Saarimäki-Vire J, et al. An activating STAT3 mutation causes neonatal diabetes through premature induction of pancreatic differentiation. Cell Rep. 2017;19(2):281-294.

49. Plasterer HL, Deutsch EC, Belmonte M, Egan E, Lynch DR, Rusche JR. Development of frataxin gene expression measures for the evaluation of experimental treatments in Friedreich's ataxia. PLoS One. 2013;8(5):e63958.

50. Guo L, et al. Liquid chromatography-high resolution mass spectrometry analysis of platelet frataxin as a protein biomarker for the rare disease Friedreich's Ataxia. Anal Chem. 2018;90(3):2216-2223.

51. Blair IA, et al. The current state of biomarker research for Friedreich's ataxia: a report from the 2018 FARA biomarker meeting Future Sci OA. 2019;5(6):FSO398.

52. Tews D, Werner U, Eckel J. Enhanced protection against cytokine- and fatty acid-induced apoptosis in pancreatic beta cells by combined treatment with glucagon-like peptide-1 receptor agonists and insulin analogues. Horm Metab Res. 2008;40(3):172-180

53. Tsunekawa S, et al. Protection of pancreatic beta-cells by exendin-4 may involve the reduction of endoplasmic reticulum stress; in vivo and in vitro studies. J Endocrinol. 2007;193(1):65-74.

54. Chen J, Couto FM, Minn AH, Shalev A. Exenatide inhibits beta-cell apoptosis by decreasing thioredoxin-interacting protein. Biochem Biophys Res Commun. 2006;346(3):1067-1074.

55. Padmasekar M, Lingwal N, Samikannu B, Chen C, Sauer H, Linn T. Exendin-4 protects hypoxic islets from oxidative stress and improves islet transplantation outcome. Endocrinology. 2013;154(4):1424-1433.

56. Fernández-Millán E, et al. Glucagon-like peptide-1 improves beta-cell antioxidant capacity via extracellular regulated kinases pathway and Nrf2 translocation. Free Radic Biol Med. 2016;95:16-26.

57. Mangmool S, Hemplueksa P, Parichatikanond W, Chattipakorn N. Epac is required for GLP-1R-mediated inhibition of oxidative stress and apoptosis in cardiomyocytes. Mol Endocrinol. 2015;29(4):583-596.

58. Schulz JB, et al. Oxidative stress in patients with Friedreich ataxia. Neurology. 2000;55(11):1719-1721.

59. Sparaco M, et al. Friedreich's ataxia: oxidative stress and cytoskeletal abnormalities. J Neurol Sci. 2009;287(1-2):111-118

60. Tanaka Y, Gleason CE, Tran PO, Harmon JS, Robertson RP. Prevention of glucose toxicity in HIT-T15 cells and Zucker diabetic fatty rats by antioxidants. Proc Natl Acad Sci USA. 1999;96(19):10857-10862.

61. Oprescu AI, et al. Free fatty acid-induced reduction in glucose-stimulated insulin secretion: evidence for a role of oxidative stress in vitro and in vivo. Diabetes. 2007;56(12):2927-2937.

62. Danielpur L, et al. GLP-1-RA Corrects mitochondrial labile iron accumulation and improves $\beta$-cell function in type 2 Wolfram syndrome. J Clin Endocrinol Metab. 2016;101(10):3592-3599.

63. Martelli A, Puccio H. Dysregulation of cellular iron metabolism in Friedreich ataxia: from primary iron-sulfur cluster deficit to mitochondrial iron accumulation. Front Pharmacol. 2014;5:130.

64. Shan Y, et al. Frataxin deficiency leads to defects in expression of antioxidants and Nrf2 expression in dorsal root ganglia of the Friedreich's ataxia YG8R mouse model. Antioxid Redox Signal. 2013;19(13):1481-1493.

65. D'Oria V, et al. Frataxin deficiency leads to reduced expression and impaired translocation of NF-E2-related factor (Nrf2) in cultured motor neurons. Int J Mol Sci. 2013;14(4):7853-7865.

66. Paupe V, et al. Impaired nuclear Nrf2 translocation undermines the oxidative stress response in Friedreich ataxia. PLoS One. 2009;4(1):e4253.

67. Petrillo S, et al. Nrf2-inducers counteract neurodegeneration in frataxin-silenced motor neurons: disclosing new therapeutic targets for Friedreich's Ataxia. Int J Mol Sci. 2017;18(10):E2173.

68. Koehler JA, et al. Glucagon-like peptide-1 receptor agonists increase pancreatic mass by induction of protein synthesis. Diabetes. 2015;64(3):1046-1056.

69. Deutsch EC, et al. A rapid, noninvasive immunoassay for frataxin: utility in assessment of Friedreich ataxia. Mol Genet Metab. 2010;101(2-3):238-245.

70. Secher A, et al. The arcuate nucleus mediates GLP-1 receptor agonist liraglutide-dependent weight loss. J Clin Invest. 2014;124(10):4473-4488.

71. Kastin AJ, Akerstrom V. Entry of exendin-4 into brain is rapid but may be limited at high doses. Int J Obes Relat Metab Disord. 2003;27(3):313-318

72. Christensen M, et al. Transfer of liraglutide from blood to cerebrospinal fluid is minimal in patients with type 2 diabetes. Int $J$ Obes (Lond). 2015;39(11):1651-1654.

73. Zhang L, Zhang L, Li L, Hölscher C. Neuroprotective effects of the novel GLP-1 long acting analogue semaglutide in the MPTP Parkinson's disease mouse model. Neuropeptides. 2018;71:70-80.

74. Tamargo IA, et al. Novel GLP-1R/GIPR co-agonist "twincretin" is neuroprotective in cell and rodent models of mild traumatic brain injury. Exp Neurol. 2017;288:176-186.

75. Cunha DA, et al. Death protein 5 and p53-upregulated modulator of apoptosis mediate the endoplasmic reticulum stress-mitochondrial dialog triggering lipotoxic rodent and human $\beta$-cell apoptosis. Diabetes. 2012;61(11):2763-2775

76. Marselli L, et al. Are we overestimating the loss of beta cells in type 2 diabetes? Diabetologia. 2014;57(2):362-365.

77. Ravassard $\mathrm{P}$, et al. A genetically engineered human pancreatic $\beta$ cell line exhibiting glucose-inducible insulin secretion. $J$ Clin Invest. 2011;121(9):3589-3597.

78. Brozzi F, et al. Cytokines induce endoplasmic reticulum stress in human, rat and mouse beta cells via different mechanisms Diabetologia. 2015;58(10):2307-2316

79. Khaldi MZ, Guiot Y, Gilon P, Henquin JC, Jonas JC. Increased glucose sensitivity of both triggering and amplifying pathways of insulin secretion in rat islets cultured for $1 \mathrm{wk}$ in high glucose. Am J Physiol Endocrinol Metab. 2004;287(2):E207-E217.

80. Marchetti P, Suleiman M, Marselli L. Organ donor pancreases for the study of human islet cell histology and pathophysiology: a precious and valuable resource. Diabetologia. 2018;61(4):770-774. 